ARTICLE

Received 16 Feb 2015 | Accepted 28 Apr 2015 | Published 17 Jun $2015 \quad$ DOl: 10.1038/ncomms8362 OPEN

\title{
NELL-1 in the treatment of osteoporotic bone loss
}

\author{
Aaron W. James ${ }^{1,2,3, \star}$, Jia Shen ${ }^{1,2, \star}$, Xinli Zhang ${ }^{2, \star}$, Greg Asatrian², Raghav Goyal ${ }^{2}$, Jin H. Kwak², Lin Jiang ${ }^{4}$, \\ Benjamin Bengs ${ }^{1}$, Cymbeline T. Culiat ${ }^{5}$, A. Simon Turner ${ }^{6}$, Howard B. Seim $1 I^{6}$, Benjamin M. Wu ${ }^{7}$, Karen Lyons ${ }^{1}$, \\ John S. Adams ${ }^{1}$, Kang Ting ${ }^{1,2} \&$ Chia Soo ${ }^{1,8}$
}

NELL-1 is a secreted, osteoinductive protein whose expression rheostatically controls skeletal ossification. Overexpression of NELL-1 results in craniosynostosis in humans and mice, whereas lack of Nell-1 expression is associated with skeletal undermineralization. Here we show that Nell-7-haploinsufficient mice have normal skeletal development but undergo age-related osteoporosis, characterized by a reduction in osteoblast:osteoclast (OB:OC) ratio and increased bone fragility. Recombinant NELL-1 binds to integrin $\beta 1$ and consequently induces $W n t / \beta$-catenin signalling, associated with increased $O B$ differentiation and inhibition of OC-directed bone resorption. Systemic delivery of NELL-1 to mice with gonadectomy-induced osteoporosis results in improved bone mineral density. When extended to a large animal model, local delivery of NELL-1 to osteoporotic sheep spine leads to significant increase in bone formation. Altogether, these findings suggest that NELL-1 deficiency plays a role in osteoporosis and demonstrate the potential utility of NELL-1 as a combination anabolic/antiosteoclastic therapeutic for bone loss.

\footnotetext{
${ }^{1}$ Department of Orthopaedic Surgery and the Orthopaedic Hospital Research Center, UCLA and Orthopaedic Hospital, University of California, Los Angeles, California 90095, USA. ${ }^{2}$ Division of Growth and Development, Section of Orthodontics, School of Dentistry, University of California, Los Angeles, California 90095, USA. ${ }^{3}$ Department of Pathology and Laboratory Medicine, David Geffen School of Medicine, University of California, Los Angeles, California 90095, USA. ${ }^{4}$ Department of Neurology, Easton Center for Alzheimer's Disease Research, Molecular Biology Institute, University of California, Los Angeles, California 90095, USA. ${ }^{5}$ Oak Ridge National Laboratory (ORNL), Oak Ridge, Tennessee 37830, USA. ${ }^{6}$ Department of Veterinary Sciences, Colorado State University, Fort Collins, Colorado 80523, USA. 7 Department of Bioengineering and Department of Material Sciences, University of California, Los Angeles, California 90095, USA. ${ }^{8}$ Division of Plastic and Reconstructive Surgery, Department of Surgery, David Geffen School of Medicine, University of California, Los Angeles, Los Angeles, California 90095, USA. * These authors contributed equally to this work. Correspondence and requests for materials should be addressed to K.T. (email: kting@dentistry.ucla.edu) or to C.S. (email: bsoo@ucla.edu).
} 
O steoporosis is a disease of severe bone loss affecting an estimated 10 million Americans and causing two million pathological fractures per year ${ }^{1}$. Osteoporosis results from an imbalance between bone formation and resorption. This balance depends on both the number of osteoblastic (OB) and osteoclastic (OC) cells as well as their cellular activity within the bone metabolic unit ${ }^{2-4}$. Typically, bone anabolic agents produce a secondary osteoclastogenic response, as is the case for $\mathrm{PTH}$, BMP2 and retinoic acid $^{5-9}$. The interest in therapies with the potential to uncouple $\mathrm{OB}$ and $\mathrm{OC}$ activity has led to intense focus on activation of the $\mathrm{Wnt} / \beta$-catenin signalling pathway, as this pathway has predominant anabolic effects on bone. However, high lipophilicity and insolubility make recombinant Wnt proteins challenging for bioactive delivery ${ }^{10-12}$. An alternative approach is to increase levels of Wnt signalling via inactivation of endogenous Wnt inhibitors (Sclerostin (SOST) and DKK-1). In fact, treatment with anti-SOST and anti-DKK-1 antibodies results in increased $\mathrm{OB}$ number and activity, reduced $\mathrm{OC}$ number and activity, and a consequent increase in bone mineral density $(\mathrm{BMD})^{13,14}$, observed in rat ${ }^{15,16}$, non-human primate ${ }^{17}$ and human studies ${ }^{18}$. In aggregate, positive regulation of the Wnt/ $\beta$ catenin signalling pathway has emerged as a promising new field for anabolic, anti-OC therapies in osteoporosis.

NELL-1 is a unique secreted protein of 810 amino acids first studied in the context of human craniofacial skeletal development, where NELL-1 was noted to be osteoinductive and its overexpression associated with human craniosynostosis (CS $)^{19}$. Since that time, transgenic Nell-1-overexpressing mice have been observed to recapitulate a CS-like phenotype ${ }^{20}$. Conversely, Nell-1-deficient mice (as developed by N-ethyl-N-nitrosourea (ENU)-induced mutagenesis) exhibit cranial and vertebral bone defects with undermineralization ${ }^{21}$. Mechanistically, NELL-1 binds to the cell surface receptor integrin $\beta 1$ (ref. 22) and regulates activity of the master osteogenic transcription factor, Runt-related transcription factor-2 (Runx2) ${ }^{23}$. Despite the welldemonstrated osteogenic effects of NELL-1 (refs 24-27), there has been little understanding of the signalling pathway through which the effects of NELL-1 are exerted, nor the potential role of NELL1 in osteoporosis. However, a recent genome-wide study of single-nucleotide polymorphisms found a linkage between NELL-1 and osteoporosis in human patients ${ }^{28}$.

Here we explore the causative and therapeutic possibilities of manipulating NELL-1 signalling in osteoporosis. First, the effects of Nell-1 deficiency in skeletal aging were evaluated by studying the skeletons of Nell-1-haploinsufficient mice. Next, the opposing effects of NELL-1 on OB and OC cells as well as mechanisms underlying NELL-1's activation of $\mathrm{Wnt} / \beta$-catenin signalling were uncovered. Further, a large animal study was performed in which surgical delivery of NELL-1 was used in order to combat osteoporotic bone loss in sheep spine. Finally, systemic administration of NELL-1 was used to reverse gonadectomy-induced osteoporotic bone loss in mice. In sum, the present study describes a new role for NELL-1 as an aetiological factor and a treatment of osteoporosis.

\section{Results}

Osteoporotic phenotype of Nell-1-haploinsufficient mouse. NELL-1 expression, known to be present during skeletal development, was evaluated with skeletal aging. Nell-1 protein showed a significant reduction with age, as shown by immunohistochemistry of trabecular bone-lining $\mathrm{OBs}$ in the rat lumbar spine (Fig. 1a). Semiquantitative analysis demonstrated a significant decrease in Nell-1 ${ }^{+}$bone-lining cells with age (Fig. 1b). These results were further quantified using quantitative reverse transcription (qRT)-PCR, showing a progressive loss of Nell-1 in the bone with age (Fig. 1c). The consequences of Nell-1 deficiency on skeletal aging were next assessed. Deficiency was induced by the generation of a point mutation in the Nell-1 gene $\left(\right.$ Nell-1 $\left.1^{6 \mathrm{R} / 6 \mathrm{R}}\right)$ resulting in a premature stop codon and near complete loss of transcript levels. Complete deficiency (Nell-1 $\left.{ }^{6 \mathrm{R} / 6 \mathrm{R}}\right)$ is neonatal lethal with major skeletal anomalies, while the heterozygote $\left(\right.$ Nell-1 $\left.1^{+/ 6 R}\right)$ mice are without gross abnormalities at birth $^{21}$. The Nell-1 $1^{+/ 6 R}$ mouse skeleton was studied across its lifetime. The neonatal (P1 days), young adult (1 month) and adult skeleton (6 months) showed no significant skeletal phenotype, as assessed by combined live $\mathrm{CT} /$ fluoride radioisotope $\left({ }^{18} \mathrm{~F}\right)$ incorporation studies, and histomorphometric analyses (Supplementary Fig. 1, Supplementary Table 1). In contrast, by 18 months of life the Nell-1 $1^{+/ 6 \mathrm{R}}$ mouse developed significant osteoporosis (Fig. 1, Supplementary Fig. 2). This was apparent both in analysis of the lumbar spine (Fig. 1d-t) and the distal femur (Supplementary Fig. 2f-k). Analyses showed significantly reduced $\mathrm{BMD}$ and reduced ${ }^{18} \mathrm{~F}$ incorporation (Fig. 1d-f). Dual energy X-ray absorptiometry (DXA), high-resolution microCT and trabecular bone analyses confirmed an osteoporotic phenotype in the aged Nell-1 $1^{+/ 6 R}$ mouse in both the lumbar spine and femur (Fig. 1g-j, Supplementary Fig. 2a-k). Further, the aged Nell-1 $1^{+/ 6 \mathrm{R}}$ bone showed increased bone fragility and decreased bone stiffness as shown by both computer-simulated biomechanical compression testing as well as microindentation testing (Supplementary Fig. 2l-r). Next, serum biomarkers evaluated total $\mathrm{OB}$ and $\mathrm{OC}$ activity (Fig. $1 \mathrm{k}-\mathrm{m}$ ). Total bone formation activity (serum Procollagen I N-terminal Propeptide (PINP)) was reduced among Nell-1 ${ }^{+/ 6 \mathrm{R}}$ mice, while total bone resorption activity was increased in Nell-1 $1^{+/ 6 \mathrm{R}}$ mice (serum Tartrate Resistant Acid Phosphatase 5b (TRAP5b) and C-Terminal Telopeptide (CTX)). Histological analysis confirmed a significant reduction in the vertebral body trabecular bone among Nell-1 $1^{+16 \mathrm{R}}$ specimens (Fig. $1 \mathrm{n}-\mathrm{q}$, Supplementary Fig. 2s-y). Next, OB and OC prevalence was analysed histologically (Fig. $1 \mathrm{r}-\mathrm{t}$ ). OB number per bone perimeter (Ob.N per B.Pm) was significantly reduced in the Nell-1 $1^{+/ 6 \mathrm{R}}$ lumbar vertebrae. Next, OC numbers, evaluated by TRAP staining, showed a significant increase in osteoclast number (Oc.N) per B.Pm among Nell-1 ${ }^{+/ 6 \mathrm{R}}$ vertebrae. Thus, by radiographic, biomechanical and histologic analyses, Nell-1 haploinsufficiency results in normal skeletogenesis and development, but results in an osteoporotic defect with age. This osteoporotic defect, manifested in both sexes, is characterized by increased cortical bone fragility, altered trabecular bone morphology, decreased $\mathrm{OB}$ and increased OC number.

NELL-1 has inverse effects on bone formation and resorption. In vivo studies demonstrated marked alterations in both $\mathrm{OB} / \mathrm{OC}$ number and activity. Next, the isolated cellular effects of Nell-1 haploinsufficiency were determined by the culture of either OB or OC precursors. First, marrow-derived Nell-1 ${ }^{+/ 6 \mathrm{R}} \mathrm{OB}$ precursors were examined (Fig. 2a-e). Consistent with in vivo findings, Nell-1 $1^{+/ 6 R}$ OB precursors showed a significant reduction in expression across all markers of in vitro osteogenic differentiation, including alkaline phosphatase (ALP)-positive cells, as well as alizarin red-positive bone nodules. Specific gene expression showed a global reduction across both 'early' and 'later' markers of $\mathrm{OB}$ differentiation. In addition, $\mathrm{OB}$ precursor proliferation was reduced among Nell-1 ${ }^{+/ 6 \mathrm{R}}$ cells. Next, converse experiments were performed with OC precursors (Fig. $2 \mathrm{f}-\mathrm{j}$ ). OC precursors from Nell-1 $1^{+/+}$and Nell-1 $1^{+/ 6 \mathrm{R}}$ aged littermates were seeded on bone discs for resorption assays. By gross photographic and scanning electron microscopy assessments, greater bone resorption was detected among Nell-1 ${ }^{+/ 6 R}$ OC precursors. These results 
a

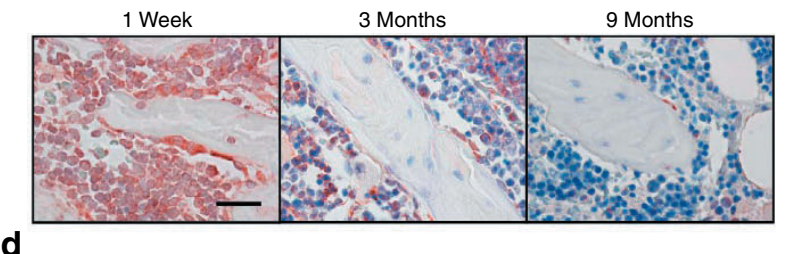

d

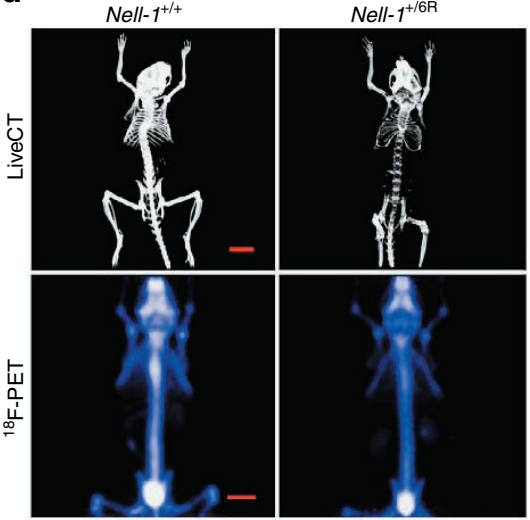

g

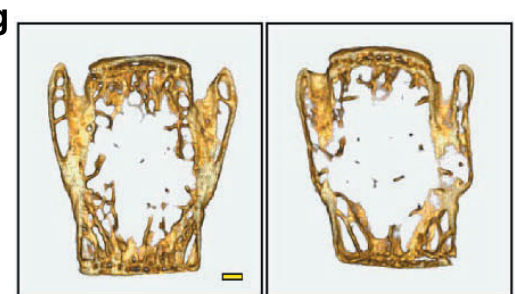

n

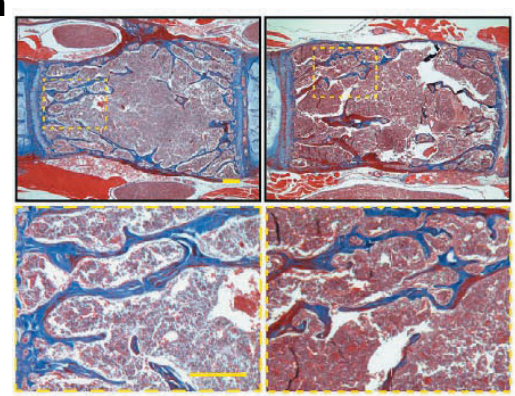

$\mathbf{r}$

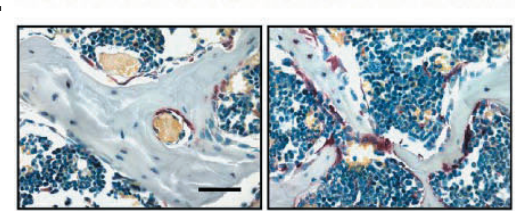

h

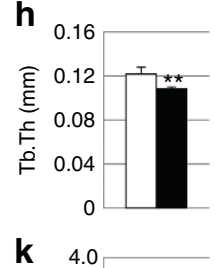

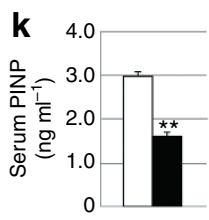
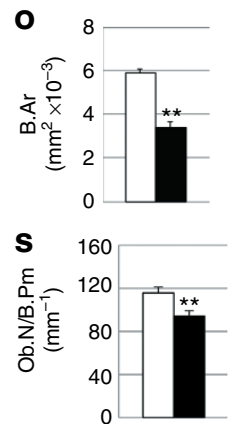

b

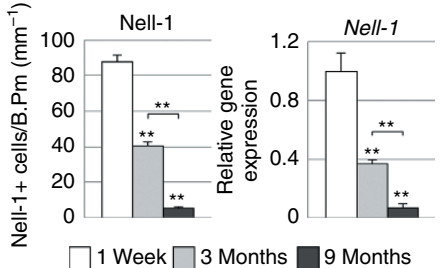

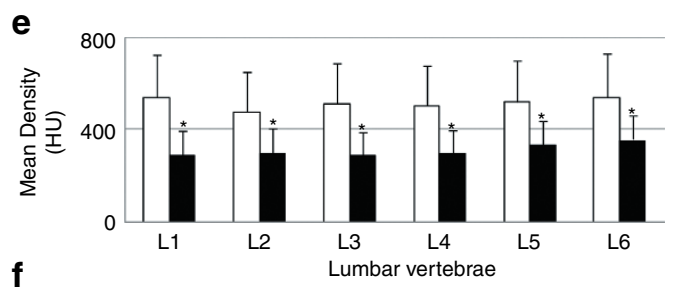

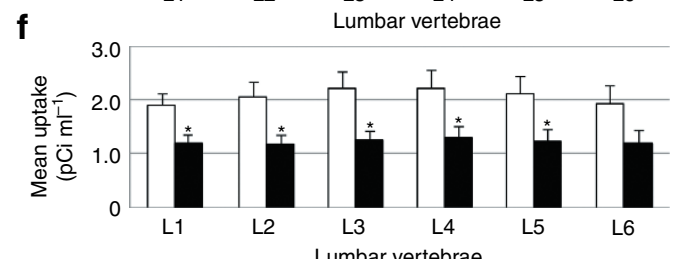

i

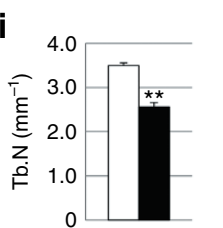

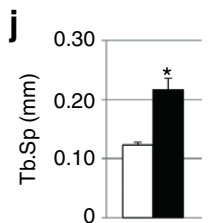

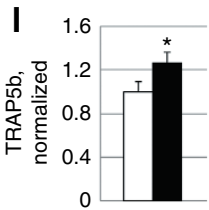

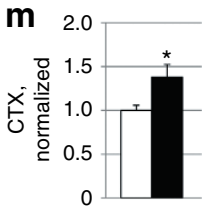

p 50

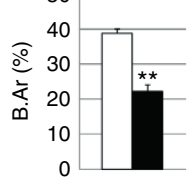

q 0.5

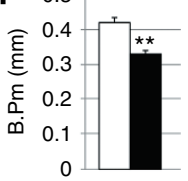

t

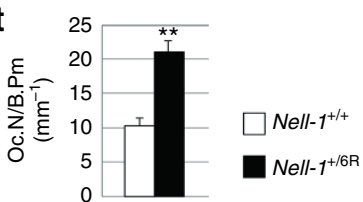

Figure 1 | Osteoporotic phenotype of aged Nell-1-haploinsufficient mice. (a) Immunohistochemical staining for Nell-1 protein in rat spines, at 1 week, and 3 and 9 months. (b) Semiquantification of Nell-1 immunohistochemical staining, expressed as Nell-1+ bone-lining cells per B.Pm ( $N=20,39$ and 25 images, respectively). (c) Relative Nell-1 gene expression in mouse spines at 1 week, and 3 and 9 months, using qRT-PCR ( $N=3$ samples per time point). (d) $3 \mathrm{D}$ image of liveCT and ${ }^{18} \mathrm{~F}$ radioisotope incorporation of aged (18-month old) wild-type $\left(\mathrm{Nell}-1^{+} /{ }^{+}\right)$and Nell-1-haploinsufficient (Nell-1+/6R) mice. (e) Quantification of BMD stratified by the lumbar vertebral level (L1-L6; $N=11$ and 12 mice, respectively). (f) Quantification of ${ }^{18} \mathrm{~F}$ incorporation ( $N=6$ mice per genotype). (g) 3D coronal reconstructions of lumbar vertebrae of aged Nell-1 ${ }^{+/+}$and Nell-1+/6R mice. (h-j) Trabecular analyses of the lumbar vertebrae, including (h) trabecular bone thickness (Tb.Th), (i) number (Tb.N) and (j) spacing (Tb.Sp; $N=12$ and 19 vertebrae, respectively). (k-m) Serum studies among Nell-1 ${ }^{+/+}$and Nell-1 ${ }^{+/ 6 R}$ aged mice including (k) serum PINP ( $N=19$ and 17 mice), (I) serum TRAP5b ( $N=10$ and 9 mice) and (m) serum CTX ( $N=12$ and 22 mice). (n) Masson's trichrome staining of lumbar vertebral bodies of Nell-1+/+ and Nell-1+/6R mice, coronal section.

(o-q) Histomorphometric quantifications of lumbar vertebral bodies. Measurements include (o) B.Ar ( $N=29$ and 26 images), (p) percentage (\%) B.Ar ( $N=29$ and 26 images) and (q) B.Pm ( $N=30$ and 29 images). ( $\boldsymbol{r}$ ) TRAP staining of lumbar vertebral bodies, indicating osteoclasts. Quantification of (s) Ob.N per B.Pm ( $N=20$ images per genotype) and (t) Oc.N per B.Pm ( $N=26$ and 39 images). Black scale bar, $25 \mu$ m; red scale bar, $10 \mathrm{~mm}$; yellow scale bar, $100 \mu \mathrm{m}$. HU: Hounsfield Units; pCi (pico Curie). Data points indicate the means, while error bars represent one s.e.m. Experiments were performed without replicate. Parametric data were analysed using an appropriate Student's $t$-test or a one-way analysis of variance (ANOVA), followed by a post hoc Tukey's test. Nonparametric data were analysed with a Mann-Whitney U-test or a Kruskal-Wallis one-way analysis. ${ }^{\star} P<0.05,{ }^{\star \star} P<0.01$. 
a

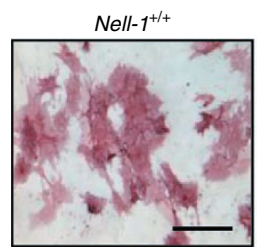

C

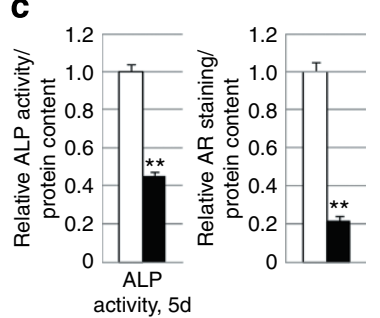

$\mathrm{Nell}-1^{+/ 6 \mathrm{R}}$

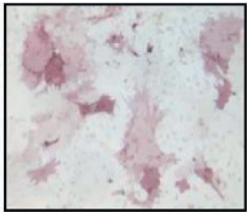

d

b

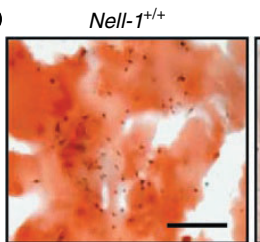

Nell-1 $1^{+/ 6 R}$

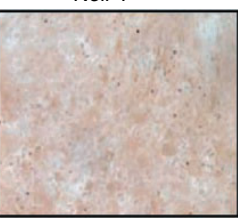

e

$\mathbf{f}$

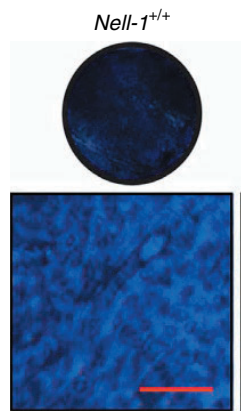

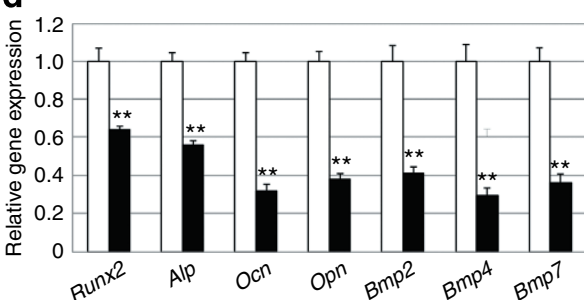

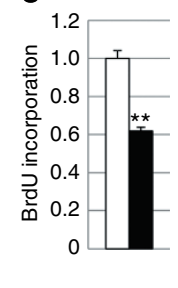

i

j
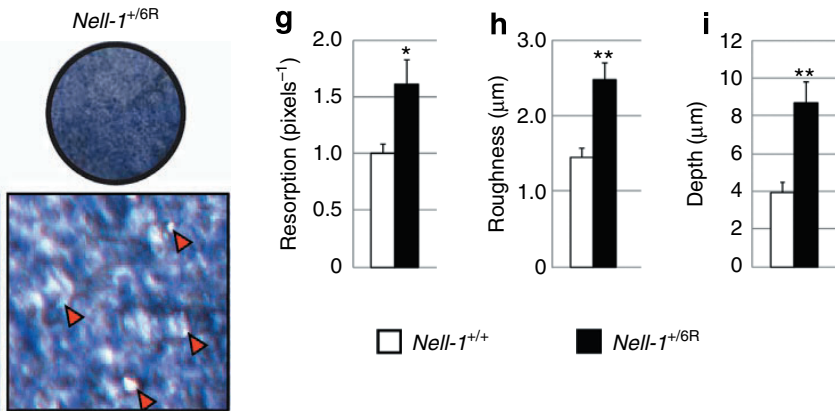

$\square \mathrm{Nell}-1^{+/+}$

Nell-1/6R

Nell-1 $1^{+/ 6 R}$

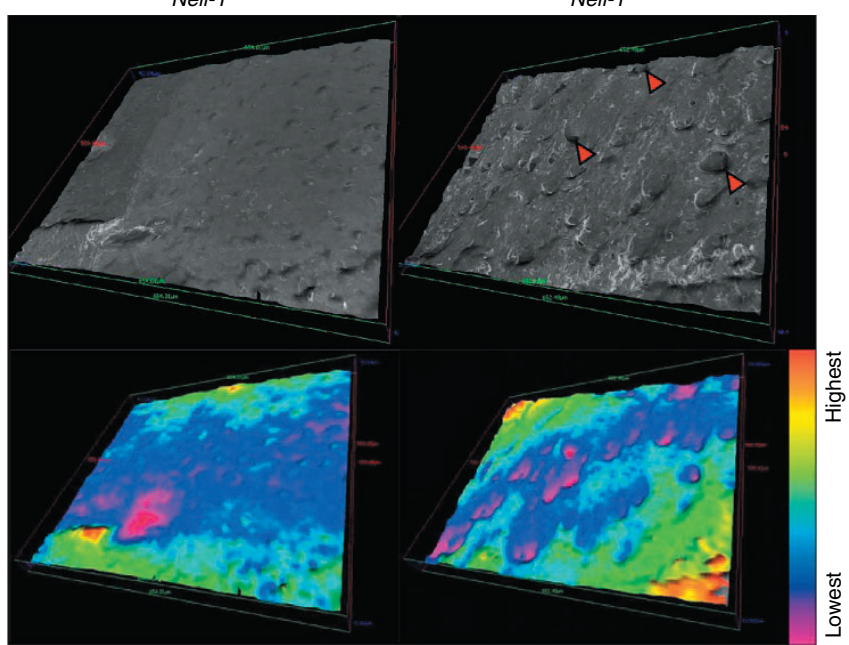

Figure 2 | Nell-1 ${ }^{+/ 6 R}$ mice exhibit impaired osteoblastic and excessive osteoclastic activity. OB or OC precursors were derived from the bone marrow of aged (18-month old) wild-type (Nell-1 ${ }^{+/+}$) and Nell-1-haploinsufficient (Nell-1+/6R) mice. (a-d) In vitro osteogenic differentiation assays of OB precursors from Nell-1 ${ }^{+/+}$and Nell-1 ${ }^{+/ 6 R}$ mice. (a) ALP staining, at 5-day osteogenic differentiation. (b) Alizarin red (AR) staining of bone nodules, at 10-day osteogenic differentiation. (c) Photometric quantification of ALP and AR activity, normalized to total protein content ( $N=3$ mice and nine wells per genotype). (d) Relative gene expression among undifferentiated Nell-1+/+ and Nell-1 ${ }^{+/ 6 R}$ OB precursors using qRT-PCR including Runx2, Alp, Osteocalcin (Ocn), Osteopontin (Opn), Bmp (Bone Morphogenetic Protein) 2, 4 and 7 ( $N=3$ mice and three wells per genotype). (e) Bromodeoxyuridine (BrdU) incorporation assays of $\mathrm{OB}$ precursors after $72-\mathrm{h}$ growth. (f-j) In vitro bone resorption assays of OC precursors derived from aged Nell-1 $+/+$ and Nell-1+/6R mice, performed in the presence of M-CSF and soluble RANKL ( $N=3$ mice and six wells per genotype). (f) Calvarial disc bone resorption assays with OC precursors from Nell-1+/+ and Nell-1+/6R mice, as shown by Toluidine blue staining after 5-day resorption. Red arrowheads indicate resorption pits. (g) Photographic quantification of bone resorption, as determined by Toluidine blue staining ( $N=3$ mice per genotype, and 9 and 11 resorption discs, respectively). (h,i) Quantification of calvarial resorption as assessed by s.e.m. of (h) the mean bone surface roughness ( $N=36$ and 29 measurements) and (i) the mean resorption pit depth ( $N=9$ measurements per genotype). (j) Reconstructions of representative resorption assays on the basis of s.e.m. Red arrows indicate representative resorption pits. Colourized s.e.m. highlights resorption pits in purple. Converse experiments in which recombinant NELL-1 is added are presented in Supplementary Fig. 3. Black scale bar, $55 \mu \mathrm{m}$; red scale bars, $20 \mu \mathrm{m}$. Data points indicate the means, while error bars represent one s.e.m. In vitro experiments were performed in biological triplicate, unless otherwise described. Parametric data were analysed using an appropriate Student's $t$-test or a one-way ANOVA, followed by a post hoc Tukey's test. Nonparametric data were analysed with a Mann-Whitney U-test or a Kruskal-Wallis one-way analysis. ${ }^{*} P<0.01$. 
a

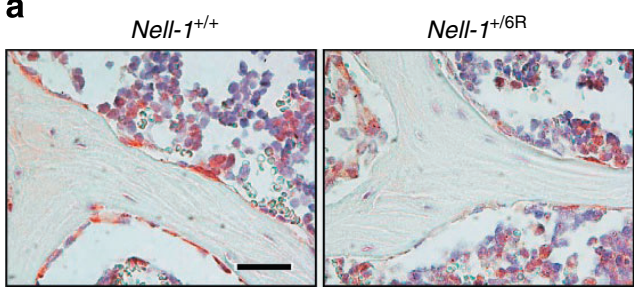

d

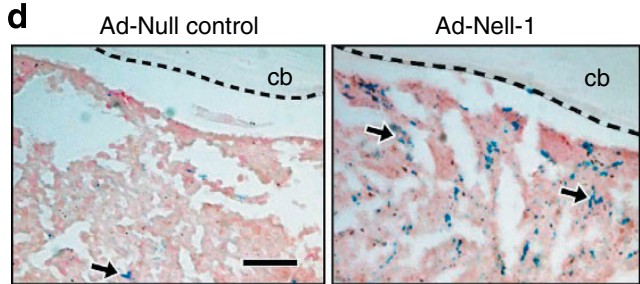

b

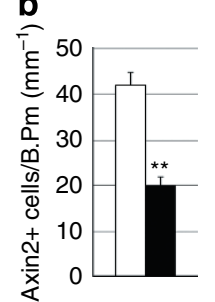

e

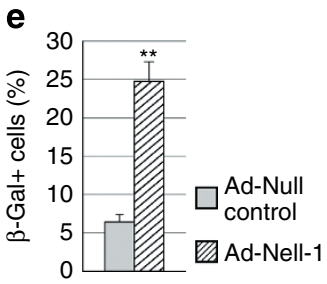

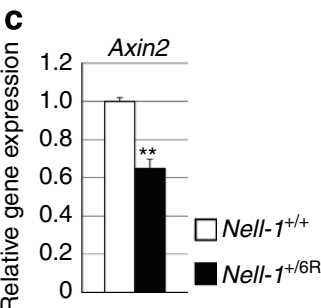

Ad-Nell-1

Figure 3 | Nell-1 signalling activates Wnt/ $\beta$-catenin signalling activity in vivo. (a-d) Evaluation of Wnt/ $\beta$-catenin signalling in Nell-1+/6R animals. (a) Axin2 immunohistochemistry in aged (18-month old) lumbar spine specimens of Nell-1+/+ and Nell-1+/6R animals, appearing brown. Haemotoxylin counterstain appears purple. (b) Semiquantification of Axin2 immunohistochemical staining among trabecular bone-lining cells ( $N=32$ and 22 images). (c) Axin2 mRNA expression in bone marrow flush of aged (18-month old) Nell-1+/+ and Nell-1+/6R mice, evaluated using qRT-PCR $(N=4$ samples per genotype). (d,e) Evaluation of Wnt/ $\beta$-catenin signalling in TOPGAL reporter mice in the context of Nell-1 overexpression. Wnt/ $\beta$-catenin signalling activity was assessed after intrafemoral injection of either Nell-1-expressing adenovirus (Ad-Nell-1 or Ad-Null control, $5 \times 10^{12} \mathrm{pt} \mathrm{ml}{ }^{-1}$ ). (d) Xgal staining in TOPgal femurs at 1 week post injection. Blue staining indicates Wnt-responsive cells, indicated by black arrows. cb: cortical bone ( $N=3$ mice per treatment group). (e) FACS analysis of femoral TOPgal bone marrow flush for $\beta$-gal positivity, indicating Wnt-responsive cells. Percentage of $\beta$-gal + cells expressed as a portion of CD45 - marrow cells (non-haematopoietic cells); analysis performed 2 weeks post Ad-Nell-1 injection ( $N=4$ mice per treatment group). Black scale bar, $25 \mu \mathrm{m}$. Data points indicate the means, while error bars represent one s.e.m. In vivo experiments were performed without replicate, unless otherwise described. Parametric data were analysed using an appropriate Student's t-test or a one-way ANOVA, followed by a post hoc Tukey's test. Nonparametric data were analysed with a Mann-Whitney U-test or a Kruskal-Wallis one-way analysis. ${ }^{\star \star} P<0.01$.

were quantified, showing an increase in resorption by Nell-1 $1^{+/ 6 \mathrm{R}}$ OC precursors (including topographic roughness and resorption pit depth). In summary, Nell-1 $1^{+/ 6 \mathrm{R}} \mathrm{OB}$ precursors showed a reduction in proliferation, differentiation and bone nodule formation. Conversely, Nell-1 $1^{+/ 6 \mathrm{R}}$ OC precursors showed increased OC activity and bone resorption when compared with Nell-1 $1^{+1+}$ cells.

Having demonstrated the effect of Nell-1 deficiency in OB and OC precursors, we next enquired as to the effects of NELL-1 gain-of-function via the addition of NELL-1 protein. To answer this, marrow-derived wild-type $\mathrm{OB}$ and $\mathrm{OC}$ precursors were harvested, and identical assays in vitro performed, now in the presence of recombinant human (rh)NELL-1 (Supplementary Fig. 3). As expected, rhNELL-1 dose-dependently increased OB precursor osteogenic differentiation by all markers. In contrast, culture of OC precursors with rhNELL-1 led to reduced bone resorption. Thus, rhNELL-1 had contrary effects on OB and OC precursors: rhNELL-1 increased $\mathrm{OB}$ precursor differentiation but inhibited OC precursor differentiation/bone resorption.

NELL-1 increases $W n t / \beta$-catenin signalling via integrin $\beta 1$. The divergent effects of NELL-1 on OB and OC cells parallel the known effects of $\mathrm{Wnt} / \beta$-catenin signalling ${ }^{29}$. To assess this link, Wnt signalling activation in the aged Nell-1 $1^{+/ 6 \mathrm{R}}$ spine was compared with Nell-1+/+ littermates (Fig. 3a-c). Axin2 immunohistochemistry was first examined as a marker of Wnt signalling activation. A significant reduction in Axin2 expression was observed among bone-lining OBs as well as in marrow cells in the Nell-1 $1^{+/ 6 \mathrm{R}}$ spine (Fig. 3a), confirmed using semiquantitative analysis of bone-lining cells (Fig. 3b). This reduction was further quantified using qRT-PCR, to reveal an $\sim 35 \%$ reduction in Axin2 expression (Fig. 3c). Next, gain-of-function experiments were performed, using a TOPGAL
Wnt reporter mouse (Fig. 3d,e). Here adenoviral Nell-1 (Ad-Nell-1) ${ }^{30}$ was injected into the femoral bone marrow cavity. In comparison with control virus, Ad-Nell-1 led to a significant increase in $\mathrm{Wnt} / \beta$-catenin signalling activity as shown by the number of $\beta$-gal ${ }^{+}$marrow cells (Fig. 3d), confirmed using flow cytometry (Fig. 3e). Thus, NELL-1 loss- or gain-of-function led to reduced or increased intramarrow $\mathrm{Wnt} / \beta$-catenin signalling, respectively.

Next, we confirmed the association between NELL-1 and Wnt/ $\beta$-catenin signalling in both OB and OC cells (Fig. 4). First, the M2-10B4 mouse bone marrow stromal cell (BMSC) line was treated with rhNELL-1 or WNT3A as a positive control. By three independent methods, rhNELL-1 induced a significant increase in Wnt/ $\beta$-catenin (Fig. $4 a-c$ ). First, immunocytochemistry for active $\beta$-catenin showed increased staining (Fig. 4a). Western blot analysis for cytoplasmic and nuclear fractions demonstrated increased nuclear $\beta$-catenin (Fig. 4b,c). Finally, M2-10B4 cells expressing the TOPFLASH reporter system exhibited an increase in TCF/LEF1 activity (Fig. 4d). Next, we determined whether interference with $\mathrm{Wnt} / \beta$-catenin signalling would impede rhNELL-1-induced OB differentiation. To answer this, two antagonists of Wnt signalling were used: DKK-1 (Fig. 4e) or XAV939 (Fig. 4f). Results showed that both Wnt antagonists inhibited rhNELL-1's induction of Runx 2 . We next determined whether rhNELL-1 induced Wnt/ $\beta$-catenin signalling in OC precursor cells, using the mouse OC line RAW264.7 (Fig. 4g-j). As assessed using immunocytochemistry, nuclear $\beta$-catenin accumulation, and gene markers of $\mathrm{Wnt} / \beta$-catenin signalling, rhNELL-1 showed a similar induction of Wnt signalling activity in RAW264.7 cells. In summary, rhNELL-1 protein activates $\mathrm{Wnt} / \beta$-catenin signalling in both $\mathrm{OB}$ and $\mathrm{OC}$ cell types in vitro. Moreover, NELL-1's induction of OB differentiation is dependent on intact $\mathrm{Wnt} / \beta$-catenin signalling.

We have previously determined that NELL-1 binds to the surface receptor integrin $\beta 1$, and that this interaction is necessary 


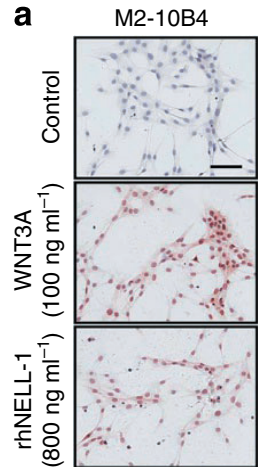

b
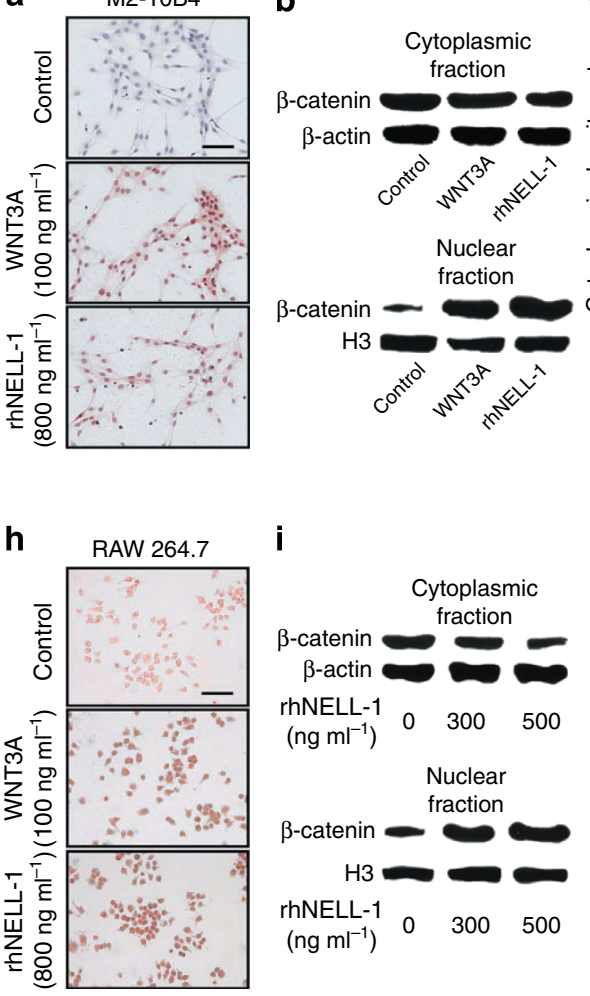

i
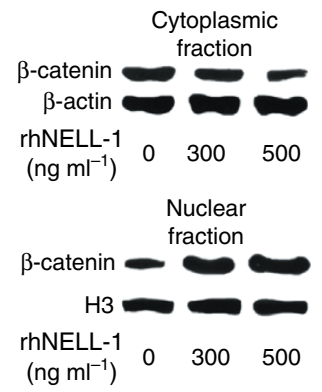

c

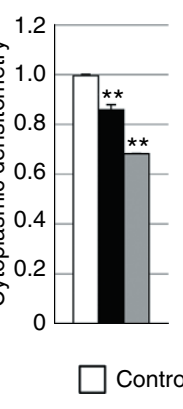

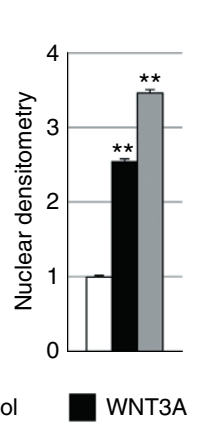

d

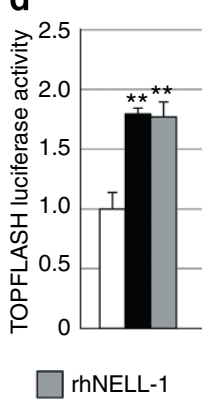

e

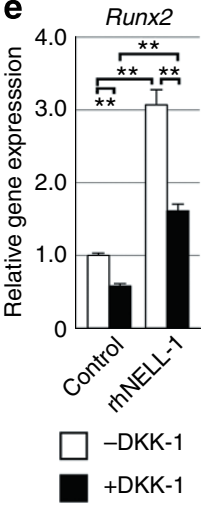

f
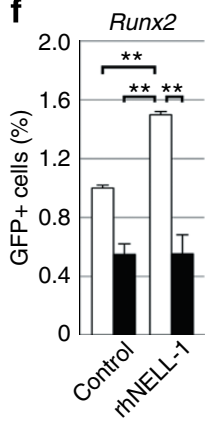

$\square$-XAV939

+XAV939 $\mathbf{g}$

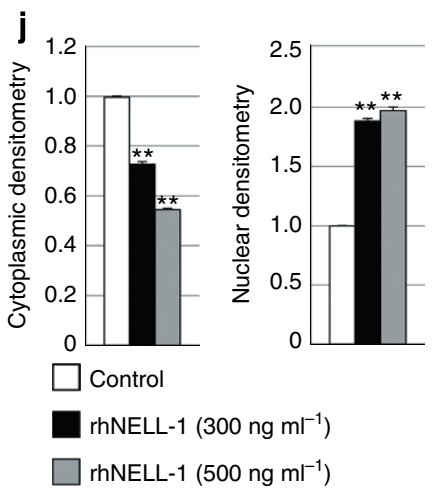

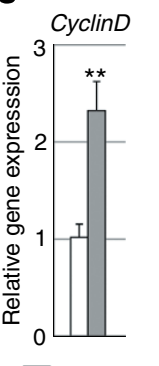

Control

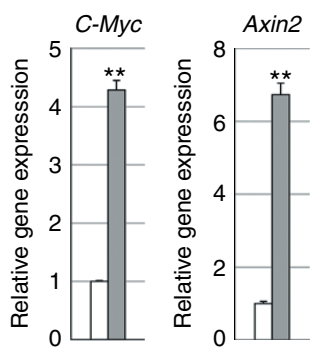

rhNELL-1
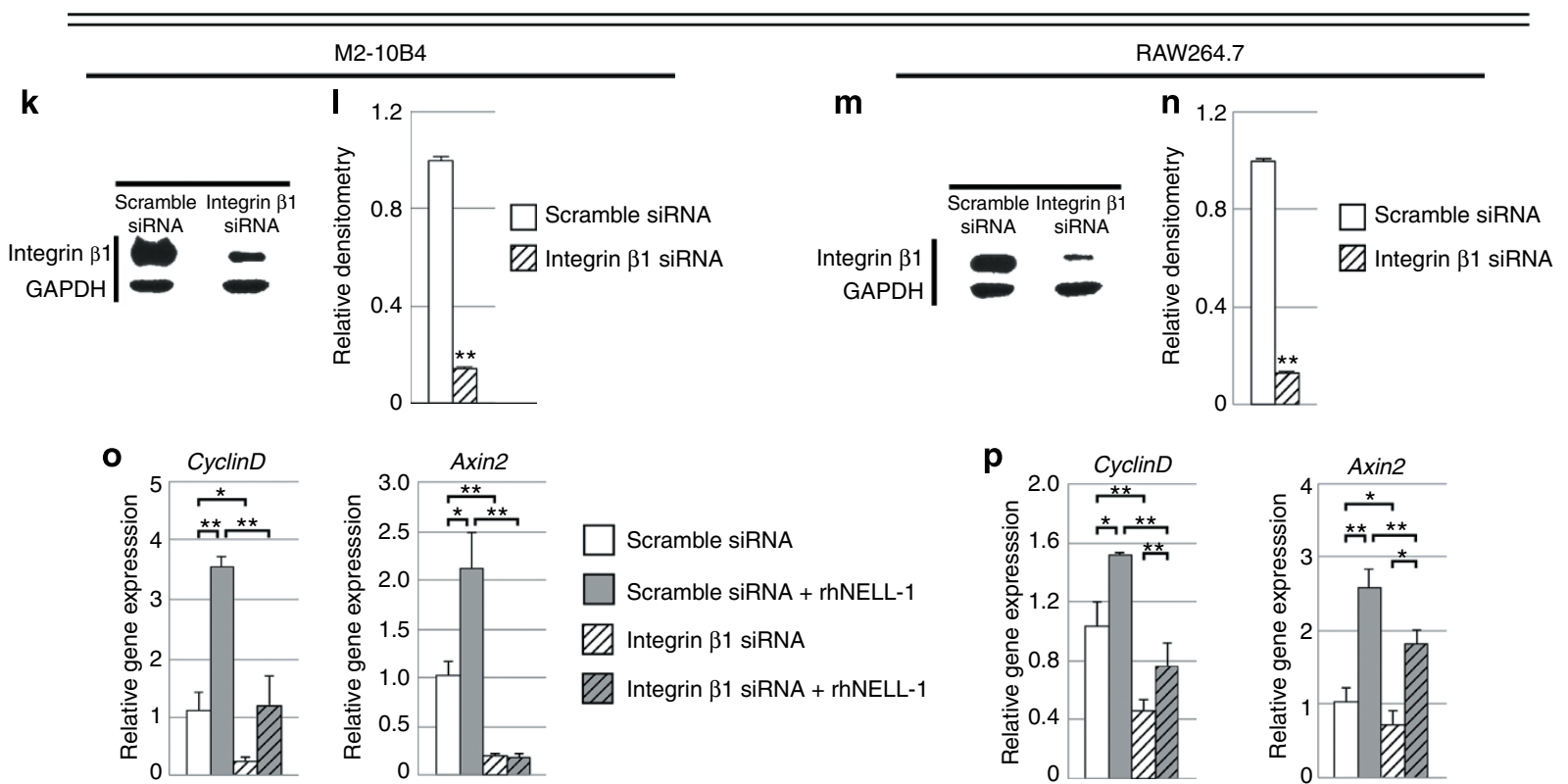

Figure 4 | NELL-1 requires integrin $\boldsymbol{\beta} 1$ to activate $\mathbf{W n t} / \boldsymbol{\beta}$-catenin signalling. (a-d) RhNELL-1 increases Wnt signalling in the M2-10B4 BMSC line. (a) Active $\beta$-catenin immunocytochemistry in M2-10B4 cells, treated with rhNELL-1, WNT3A or control (PBS). (b,c) Western blot analysis and quantification of cytoplasmic and nuclear $\beta$-catenin $(N=3$ wells per treatment). (d) M2-10B4 cells were transfected with TOPFLASH reporter $(N=4$ wells per treatment). (e,f) RhNELL-1 requires intact Wnt/ $\beta$-catenin signalling for induction of OB differentiation $(N=4$ wells per treatment). (e) M2-10B4 cells were treated with PBS or rhNELL-1 with or without DKK-1 for 3 days. Runx2 expression measured using qRT-PCR. (f) M2-10B4 cells were transduced with Runx2-EGFP reporter lentivirus and treated with PBS or rhNELL-1 with or without XAV939. Runx2 reporter assay was performed after 3 days. (g-i) RhNELL-1 increases Wnt signalling in the RAW264.7 osteoclast cell line ( $N=3$ wells per treatment). (g) Gene expression after 2 days with or without rhNELL-1. (h) Active $\beta$-catenin immunocytochemistry in RAW264.7 cells, treated with rhNELL-1, WNT3A or control (PBS). (i,j) Western blot and quantification with or without rhNELL-1. (k-p) RhNELL-1 requires integrin $\beta 1$ to activate Wnt/ $\beta$-catenin signalling ( $N=3$ wells per treatment). (k, $\mathbf{l})$ siRNA-mediated knockdown of the known NELL-1 receptor integrin $\beta 1$ was performed in M2-10B4 cells, confirmed by western blot analysis and quantification. (m,n) Similar siRNA-mediated knockdown of integrin $\beta 1$ was performed in RAW264.7 OC cells. (o,p) After 2 days of rhNELL-1 $\left(300 \mathrm{ng} \mathrm{ml}^{-1}\right)$ treatment, Wnt signalling gene expression was evaluated in either scramble or integrin $\beta 1$ siRNA-treated M2-10B4 or RAW264.7 cells. Quantitative RT-PCR for CyclinD and Axin2 was performed. Black scale bars, $100 \mu \mathrm{m}$. Data points indicate the means, while error bars represent one s.e.m. In vitro experiments were performed in biological triplicate, unless otherwise described. Parametric data were analysed using an appropriate Student's $t$-test or a one-way ANOVA, followed by a post hoc Tukey's test. Nonparametric data were analysed with a Mann-Whitney U-test or a Kruskal-Wallis one-way analysis. ${ }^{\star} P<0.05,{ }^{\star \star} P<0.01$. 
for NELL-1's positive regulation of $\mathrm{OB}$ proliferation and adhesion $^{22}$. Hasebe et al. ${ }^{31}$ subsequently verified that NELL-1 binds to integrin $\beta 1$ and integrin $\alpha 3$. Here we confirmed these findings by thermal shift assays using integrin $\alpha 3 \beta 1$ with or without NELL-1 (Supplementary Fig. 4). In order to predict potential binding sites between NELL- 1 and integrin $\beta 1$, we next employed computational modelling to determine likely sites of protein interaction (Supplementary Fig. $4 \mathrm{~b}-\mathrm{d}$ ). Integrins are $\alpha / \beta$ heterodimeric cell surface receptors that control numerous cellular processes, including $\mathrm{OB}$ and $\mathrm{OC}$ proliferation, differentiation and activity ${ }^{32}$. Furthermore, integrin receptor complexes containing integrin $\beta 1$ activate $\mathrm{Wnt} / \beta$-catenin signalling via integrin-linked kinase signalling and modulation of Glycogen synthase kinase 3 beta (GSK3 $\beta$ ) phosphorylation ${ }^{33}$. Therefore, we assayed whether integrin $\beta 1$ was required for NELL-1 activation of Wnt/ $\beta$-catenin signalling (Fig. 4k-p). Small interfering RNA (siRNA)-mediated knockdown of integrin $\beta 1$ inhibited rhNELL-1's activation of Wnt/ $\beta$-catenin signalling, both in $\mathrm{OB}$ and $\mathrm{OC}$ precursor cell lines. Thus, integrin $\beta 1$ is required for NELL-1 activation of Wnt/ $\beta$-catenin signalling in both $\mathrm{OB}$ and OC cell types.

Subsequently, we sought to extend these findings to the human patient. For this purpose, human (h)BMSCs were harvested from patients with or without a clinical history of osteoporosis (Supplementary Table 2, Supplementary Fig. 5). NELL-1 signalling was manipulated by adenoviral Nell-1 (Ad-Nell-1) or control (Ad-GFP). Ad-Nell-1 increased OB differentiation in hBMSC derived from either osteoporotic or nonosteoporotic samples. Further, Ad-Nell-1 treatment resulted in increased Wnt/ $\beta$ catenin signalling activity in nonosteoporotic and osteoporotic hBMSC, as shown by gene markers and nuclear accumulation of $\beta$-catenin.

In summary, NELL- 1 activates $\mathrm{Wnt} / \beta$-catenin signalling in $\mathrm{OB}$ and $\mathrm{OC}$ precursor cells, in a process requiring integrin $\beta 1$. Moreover, NELL-1 signalling activates Wnt $/ \beta$-catenin signalling in human cells, from either nonosteoporotic or osteoporotic patients.

NELL-1 increases bone formation in osteoporotic sheep. To translate NELL-1's osteogenic function into a clinically relevant large animal model, local surgical delivery of rhNELL-1 was performed in the sheep spine, which have similar dimensions, mineral content and collagen composition to that of humans ${ }^{34-37}$. Induction of osteoporosis was achieved using ovariectomy (OVX), glucocorticoid administration and a low-calcium and low-vitamin D diet (Supplementary Fig. 6a). Similar to human osteoporosis, lumber spines are significantly compromised and are prone to compression fracture. Local surgical delivery of rhNELL-1 was performed to L1, 3 and 5. rhNELL-1 protein was injected into the cancellous bone of the vertebral body, after lyophilization on $\beta$ tricalcium phosphate and using a hyaluronic acid carrier (Supplementary Table 3 for injection composition).

Live CT scans performed monthly after rhNELL-1 injection showed a significant increase in BMD and bone volume in rhNELL-1-treated vertebrae (Fig. 5a,b). Moreover, high-resolution microCT imaging and quantification showed increased Cortical bone Thickness (Ct.Th) and increased trabecular bone density in rhNELL-1-treated vertebrae (Fig. $5 \mathrm{c}-\mathrm{g}$ ). Histological examination confirmed a significant anabolic response to rhNELL-1 injection (Fig. 5h), quantified by histomorphometric analysis of cortical and trabecular bone measurements in the periinjection area (Supplementary Table 4). Bone distant from the injection site was analysed, showing a similar anabolic response (Supplementary Fig. 6d-g). We next examined the effects of rhNELL-1 on OB and OC number. Consistent with our in vitro observations, rhNELL-1 significantly increased Ob.N and either reduced or had no effect on Oc.N (Fig. 5i,j). In summary, local rhNELL-1 delivery had significant and sustained bone-forming effects in osteoporotic sheep, observed in both cortical and cancellous bone, and accompanied by an increased OB:OC ratio.

Systemic NELL-1 increases bone formation. While the local effects of rhNELL-1 on bone formation have been established in other models ${ }^{24-27}$, the effects of systemic rhNELL-1 administration are entirely unknown and represent a broader impact for the treatment of osteoporosis. Systemic delivery was achieved by intravenous injection of rhNELL-1 in either nonosteoporotic or OVX-induced osteoporotic mice (Fig. 6, Supplementary Fig. 7). As expected, OVX induced a loss in the mean BMD, observed over a 5-week period (Fig. 6a). Next, we examined the pharmacokinetics of systemic rhNELL-1 (Supplementary Table 5). The elimination half-life of systemic rhNELL-1 was found to be $5.52 \mathrm{~h}$ (Supplementary Fig. 7a); thus, for sustained treatment rhNELL-1 was injected every $48 \mathrm{~h}$. In comparison with control and over a 4-week period, results showed that systemic rhNELL-1 treatment induced significant bone formation in both non-OVX and OVX mice (Fig. 6b-n). Analysis of the lumbar vertebrae showed rhNELL-1-induced bone formation by weekly DXA (Fig. 6b,c), live $\mathrm{CT} /{ }^{18} \mathrm{~F}-\mathrm{PET}$ imaging (Fig. 6d) and high-resolution microCT reconstruction and quantification (Fig. 6d-j). Histologic analyses were performed, including assessment of $\mathrm{OB}$ and $\mathrm{OC}$ markers (Fig. 6k,l and Supplementary Fig. $7 \mathrm{~d}-\mathrm{g}$ ). In agreement with our prior in vitro and in vivo observations, systemic rhNELL-1 administration showed both pro-OB and anti-OC effects, with an increase in Ob.N and reduction in Oc.N. This was accompanied by reduced RANKL and increased osteoprotegerin (OPG) protein expression with rhNELL-1 administration, as observed using immunohistochemistry (Supplementary Fig. 7f,g). Bone labelling and dynamic histomorphometric analyses demonstrated a significant increase in mineral apposition rate and bone formation rate (BFR) with rhNELL-1 administration (Fig. 6m). Moreover, computer-simulated biomechanical (FEA) testing demonstrated increased bone strength with rhNELL-1 treatment, in both sham and OVX conditions (Fig. 6n). Importantly, no adverse effects were observed with rhNELL-1 in animal morbidity or mortality across the study period. As an external validity, formal 5-day repeat intravenous toxicity testing in mice showed no adverse effects (Supplementary Tables 9 and10; performed by Pacific Bioloab, Hercules, CA). Thus, NELL-1, an osteoinductive protein that activates $W n t / \beta$-catenin signalling via integrin $\beta 1$, can be a safe, systemic therapy to improve osteoporotic bone quality, tipping the balance in favour of bone anabolism over bone resorption.

\section{Discussion}

In summary, we report that Nell-1 deficiency in aged mice results in an osteoporotic phenotype, characterized by increased bone fragility, reduced Wnt/ $\beta$-catenin signalling and reduced OB:OC ratio in terms of cell number and activity. On the cellular level, NELL-1 exerts its effects through interaction with integrin $\beta 1$, and subsequent positive regulation of $\mathrm{Wnt} / \beta$-catenin signalling. Finally, rhNELL-1 increases endocortical and cancellous bone in osteoporotic animal models, via either local or systemic administration. We conclude that NELL-1 activates Wnt/ $\beta$ catenin signalling via integrin $\beta 1$, uncouples OB:OC activity, may play a protective role against bone loss and represents a new anabolic and anti-OC pharmacotherapeutic agent.

First, we have found that NELL-1 differs from most other osteoinductive molecules in its negative effects on OC bone 
a

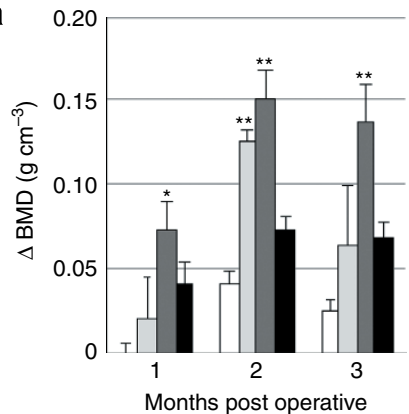

C

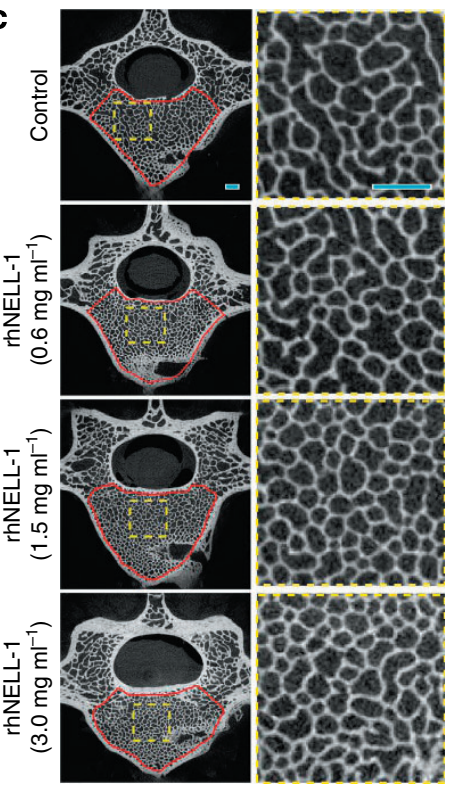

h

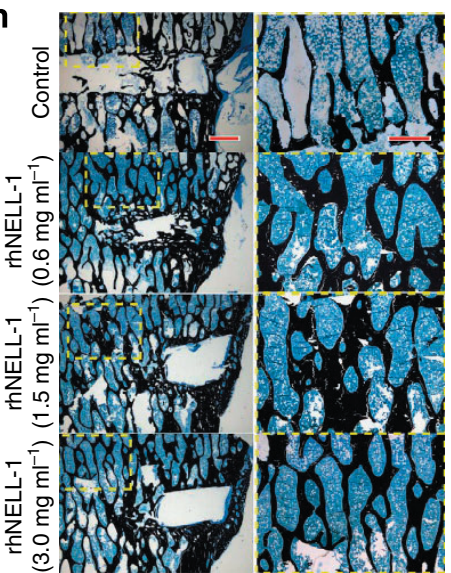

b

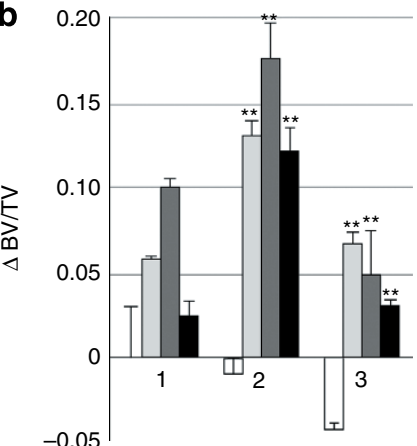

$-0.05$

Months post operative
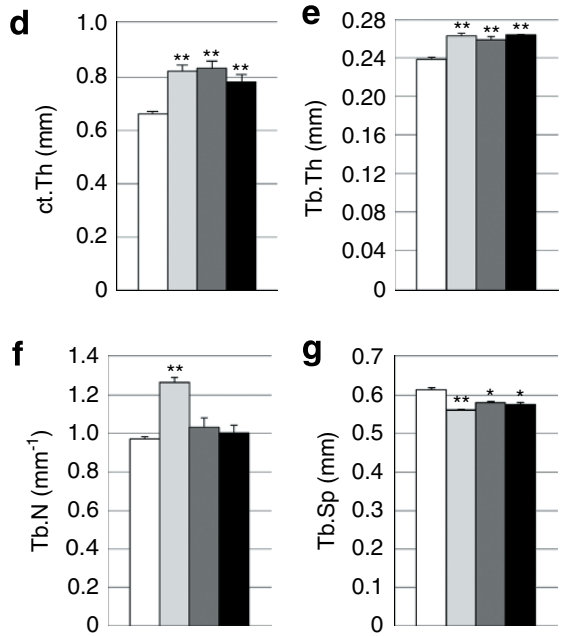

g $\quad 0.7$

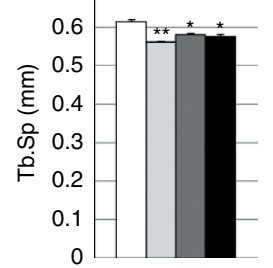

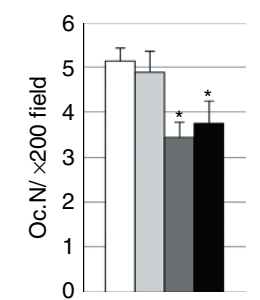

$\square$ Control

rhNELL-1

$\left(1.5 \mathrm{mg} \mathrm{ml}^{-1}\right)$

$\square^{\text {rhNELL-1 }}$

$\left(0.6 \mathrm{mg} \mathrm{ml}^{-1}\right)$

rhNELL-1

$\left(3.0 \mathrm{mg} \mathrm{ml}^{-1}\right.$ )

Figure 5 | RhNELL-1 intravertebral injection increases bone formation in osteoporotic sheep. Recombinant human (rh)NELL-1 protein or vehicle control was injected into the lumbar vertebral bodies of osteoporotic sheep. RhNELL-1 was lyophilized on $\beta$-tricalcium phosphate ( $\beta$-TCP) particles, which has been previously shown to increase the stability of rhNELL-1 in vivo ${ }^{25}$. Composition of the injected materials can be found in Supplementary Table 3 . (a) Absolute change in trabecular BMD among control- and rhNELL-1-treated vertebrae by CT quantification of individual vertebral bodies $(N=9$ control-treated vertebrae, $N=3$ vertebrae per treatment dosage). (b) Percent change in the bone volume/tissue volume (BV/TV) among control- and rhNELL-1-treated vertebrae by CT quantification of individual vertebral bodies. All quantified values are normalized to month 1, control treatment data ( $N=9$ control-treated vertebrae, $N=3$ vertebrae per treatment dose). (c) Axial sections of high-resolution $C T$ images. Yellow dashed boxes indicate the area of high magnification on right. Red outlines indicate ROI for trabecular analyses. (d) Ct.Th as determined with microCT quantification, using the cortex ipisilateral to rhNELL-1 injection ( $N=162$ control-treated measurements, $N=54$ measurements per treatment dose). (e-g) Trabecular analyses, including Tb.Th, Tb.N and Tb.Sp ( $N=9$ control-treated vertebrae, $N=3$ vertebrae per treatment dose). (h) Representative images of Von Kossa-MacNeal's Tetrachrome (VKMT) staining trabecular bone adjacent to the injection tract. Yellow dashed boxes indicate the area of high magnification on right. (i) Ob.N per B.Pm ( $N=10,9,9$ and 9 images, respectively). (j) Oc.N per $\times 200$ field $(\mathrm{N}=9,10,9$ and 9 images, respectively). Red scale bars, $0.5 \mathrm{~mm}$; blue scale bars, $1.0 \mathrm{~mm}$. Data points indicate the means, while error bars represent one s.e.m. In vivo experiments were performed without replicate, unless otherwise described. Parametric data were analysed using an appropriate Student's $t$-test or a one-way ANOVA, followed by a post hoc Tukey's test. Nonparametric data were analysed with a Mann-Whitney $U$-test or a Kruskal-Wallis one-way analysis. ${ }^{\star} P<0.05$, ${ }^{\star \star} P<0.01$ in comparison with vehicle control. See Supplementary Fig. 6 for additional details of sheep experimentation and analyses. 
a

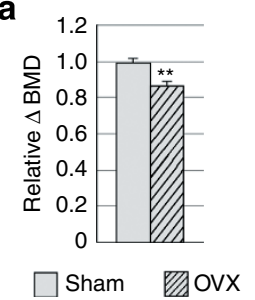

b

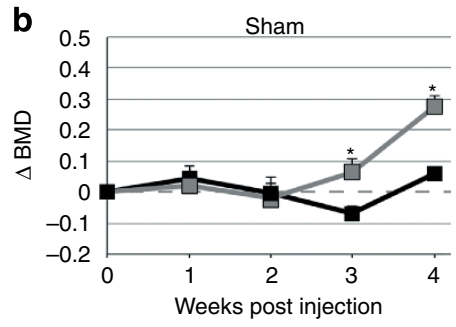

c

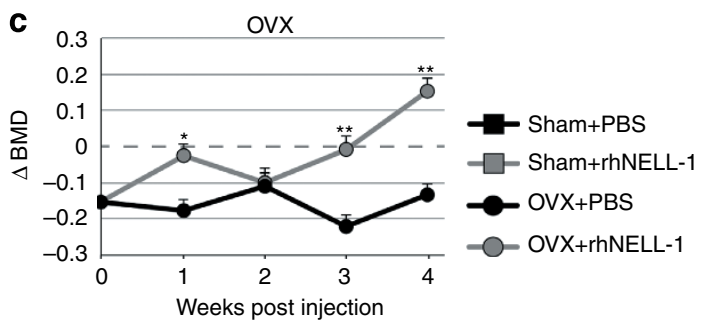

d

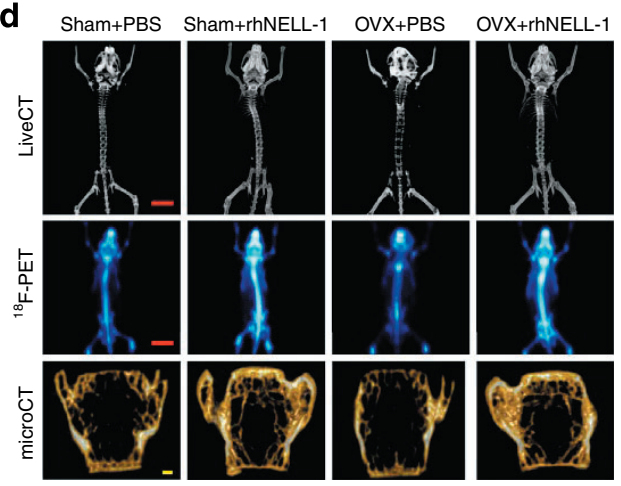

e

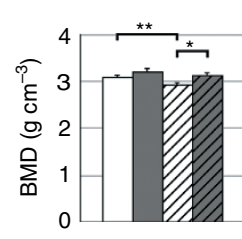

f 2
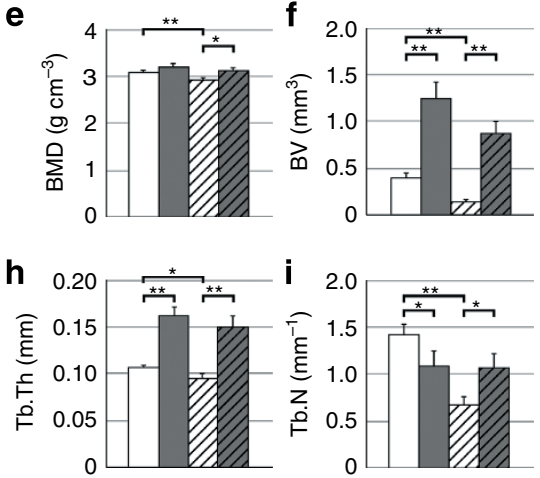
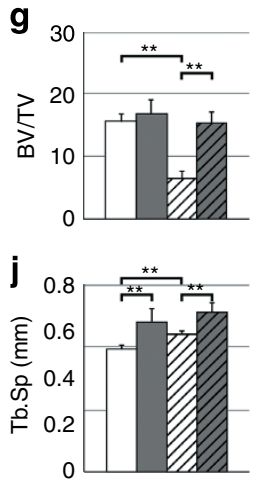

$\square$ Sham+PBS

$\mathbb{Z} \mathrm{OVX}+\mathrm{PBS}$ Sham+rhNELL-1 7 OVX+rhNELL-1
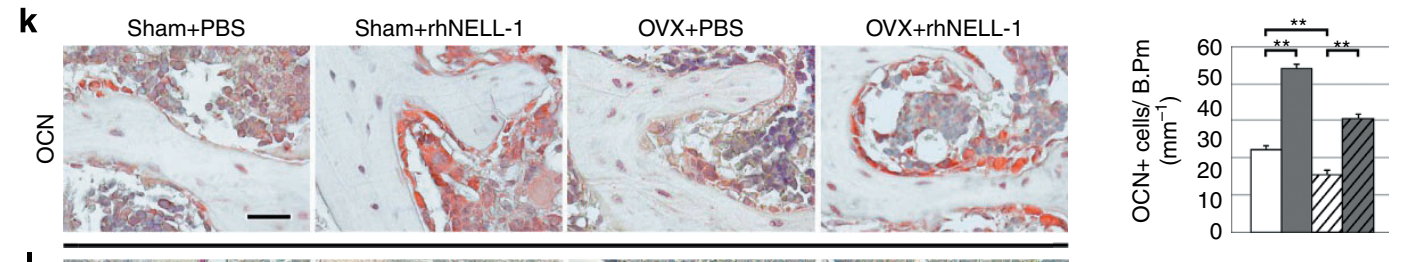

I
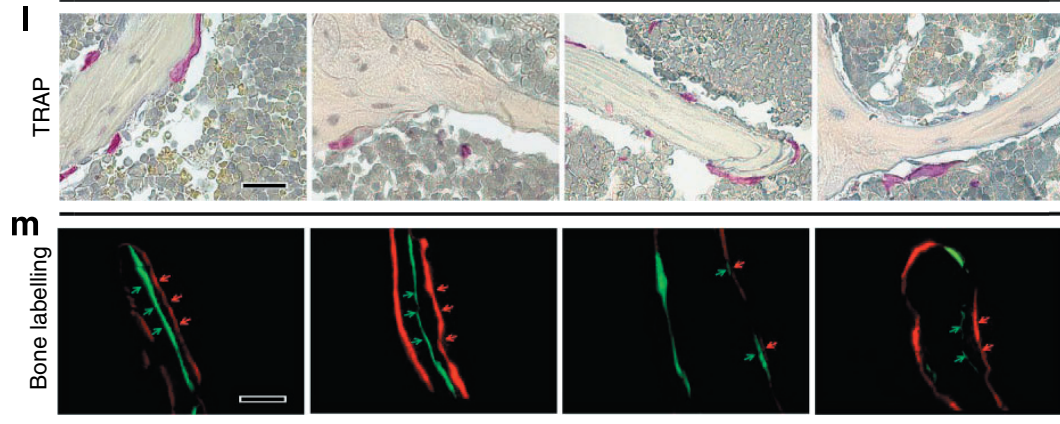

n
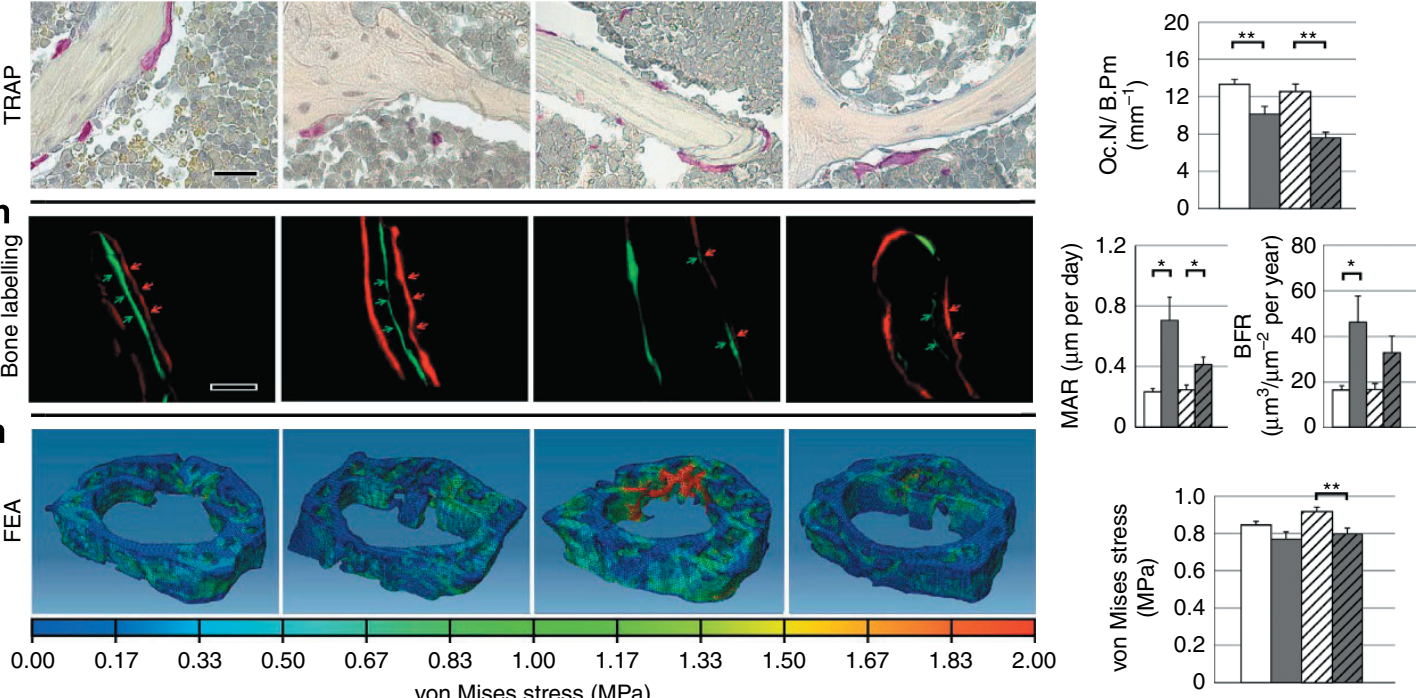

Figure 6 | RhNELL-1 intravenous injection increases bone formation in osteoporotic mice. (a) Induction of osteoporosis was performed by OVX and ensuing bone loss over a 5-week period, confirmed by DXA analysis of the lumbar vertebrae $(N=13$ and 15 mice, respectively). Confirmation of OVX was also performed post mortem by demonstration of uterine atrophy (Supplementary Fig. 7b,c). (b,c) RhNELL-1 was next administered by tail vein injection $\left(1.25 \mathrm{mg} \mathrm{kg}^{-1} \mathrm{q} 48 \mathrm{hr}\right)$ over a period of 4 weeks, and compared with PBS control treatment $(\mathrm{N}=6,7,6$ and 9 mice, respectively). DXA analysis of the lumbar vertebrae was performed weekly. (d) Representative images after 4 weeks of rhNELL-1 treatment, including live CT, ${ }^{18} \mathrm{~F}-\mathrm{PET}$ and microCT. MicroCT indices have been compared with the published norms to ensure accuracy of analysis and reporting ${ }^{59-61}$. (e-i) MicroCT quantification after 4-week treatment, including (e) BMD, (f) BV, (g) BV/TV, (h) Tb.Th, (i) Tb.N and (j) Tb.Sp ( N=6, 7, 6 and 9 mice, respectively). (k) Osteocalcin (OCN) immunohistochemical staining, and quantification of $O C N+$ bone-lining cells per B.Pm $(N=18,23,25$ and 30 images, respectively). (I) TRAP staining and quantification of TRAP +, multinucleated, bone-lining cells per B.Pm $(N=28,34,28$ and 40 images, respectively). (m) Calcein/Alizarin red complex on bone labelling and quantification of mineral apposition rate (MAR) and BFR. Red and green arrows highlight the space between fluorochrome labels $(N=3$ mice and 6 measurement fields per treatment group). (n) FEA and quantification of von Mises stress. Red colour indicates areas of high stress ( $N=6$ per treatment group). Black scale bars, $25 \mu \mathrm{m}$; red scale bar, $10 \mathrm{~mm}$; yellow scale bar, $100 \mu \mathrm{m}$. Data points indicate the means, while error bars represent one s.e.m. In vivo experiments were performed without replicate, unless otherwise described. Parametric data were analysed using an appropriate Student's $t$-test or a one-way ANOVA, followed by a post hoc Tukey's test. Nonparametric data were analysed with a Mann-Whitney U-test or a Kruskal-Wallis one-way analysis. ${ }^{\star} P<0.05,{ }^{\star \star} P<0.01$ in comparison with PBS control, unless otherwise indicated. 
resorption. Typically, anabolic agents also produce a secondary osteoclastogenic response. In contrast, we observed NELL-1 to have inhibitory effects on OCs, with decreased OC number and activity-much like anti-Wnt inhibitor therapies. Our studies suggest that NELL-1's OC inhibition may be direct and/or indirect. NELL- 1 directly activates $\mathrm{Wnt} / \beta$-catenin signalling in both $\mathrm{OB}$ and $\mathrm{OC}$ cell types. Wnt/ $\beta$-catenin signalling in $\mathrm{OBs}$ is well described to induce OB-derived expression of the major OC inhibitor OPG, and alteration of the balance between OPG and RANKL, thereby indirectly inhibiting OC activity ${ }^{38-41}$. In addition, $\mathrm{Wnt} / \beta$-catenin signalling activation in $\mathrm{OC}$ precursors has antiosteoclastogenic effects, independent of OB-elaborated $\mathrm{OPG}^{42}$. Therefore, NELL-1's positive effects on bone formation and negative effects on bone resorption may be explained both by activation of $\mathrm{Wnt} / \beta$-catenin signalling in $\mathrm{OB}$ and $\mathrm{OC}$ precursors, respectively. The extent to which the anti-OC effects of NELL-1 are similar or different to anti-Wnt inhibitor therapies, such as anti-SOST antibodies, is yet to be determined.

Second, we have shown that NELL-1 activation of $\mathrm{Wnt} / \beta$ catenin in $\mathrm{OB}$ and $\mathrm{OC}$ cell types requires expression of integrin $\beta 1$. Our research group recently identified integrin $\beta 1$ as the only known cell surface receptor for NELL-1, and that NELL-1's effects on $\mathrm{OB}$ attachment required integrin $\beta 1$ expression $^{22}$. Another research group subsequently verified that the C-terminal region of NELL-1 binds to integrin $\alpha 3 \beta 1$ (ref. 31). Integrins have documented roles in $\mathrm{OB}$ cell attachment, proliferation and differentiation ${ }^{43,44}$ and, likewise, known functions in OC adhesion and regulation of cytoskeletal organization for OC-resorptive function ${ }^{45}$. In particular, integrin $\alpha v \beta 3$ is highly expressed in OC and mediates bone extracellular matrix attachment ${ }^{45}$. Although the specific effects of integrin $\beta 1$ agonists have not been examined, integrin $\beta 1$ ablation led to reduced OC-resorptive capacity ${ }^{46}$. Our findings of NELL-1 activation of $\mathrm{Wnt} / \beta$-catenin signalling in OCs via integrin $\beta 1$ may represent a new and unexplored function of integrins in $\mathrm{OCs}^{33}$. In summary, our observations of NELL-1 interacting with integrin $\beta 1$ to exert its cellular and tissue-level effects are overall in agreement with the known roles of integrin $\beta 1$ in bone biology.

Finally, we have found that NELL-1 has potential dual uses as both a local bone-forming growth factor as well as a systemic osteogenic factor for osteoporosis. The present study advances our knowledge of the local bone-forming effects of NELL-1 to a sheep osteoporotic model. Therapeutic options for bone graft in the osteoporotic patient are limited. For example, autograft bone is less effective ${ }^{47}$ and donor-site fracture is more common ${ }^{48}$. With similar drawbacks, BMP2-based substitutes induce direct stimulation of bone resorption ${ }^{49,50}$, leading to vertebral subsidence or collapse ${ }^{51}$. With these limitations of current bone graft substitutes in the osteoporotic population, NELL-1 may be a future bone graft substitute well suited for the osteoporotic patient.

We have also demonstrated the potential therapeutic application of systemic NELL-1 delivery for the reversal of osteoporosis. NELL-1 possesses several practical and theoretical benefits over other systemic therapies for osteoporosis. First, as we observed, NELL-1 has dual anabolic and anti-OC properties. Second, NELL-1 has documented tumour-suppressive properties and its expression is lost in several carcinomas ${ }^{52,53}$. This lies in contrast to PTH, whose clinical use is limited by the risk of osteosarcoma as suggested by rat studies ${ }^{54}$. Finally, NELL-1 has an excellent safety profile. Mice with constitutive Nell-1 overexpression have a normal lifespan, and are without abnormalities excepting the skeleton ${ }^{20}$. Similarly, formal intravenous NELL-1 toxicity testing found no pathologic or biochemical abnormalities. Although the kinetics of unmodified NELL-1 protein involve rapid elimination, structural modification of NELL-1 may allow for sustained therapeutic serum levels. However, NELL-1 has known functions in neurogenesis ${ }^{55}$, chondrogenesis ${ }^{56}$ and vasculogenesis ${ }^{57}$-and a thorough study of these off-bone effects must be instituted before consideration of NELL-1 as an osteoporotic therapy.

In summary, we demonstrate that NELL-1 deficiency induces age-related osteoporosis in a rodent model. Mechanistically, our data suggest that NELL-1 modulates $O B$ and $O C$ activities through Integrin $\beta 1$-binding and subsequent $\mathrm{Wnt} / \beta$-catenin signalling activation. Most importantly, the therapeutic impact of our large and small osteoporotic animal studies demonstrates how NELL-1 may be used as a new anabolic, anti-OC treatment for osteoporosis in both local and systemic intervention. Finally, demonstration of the ability of systemic NELL-1 to activate Wnt/ $\beta$-catenin signalling will provide fundamental insights relevant to the development of NELL-1-based therapies for the treatment of multiple bone pathologies.

\section{Methods}

Animal care. All animals were cared for according to the institutional guidelines set by the Chancellor's Animal Research Committee of the Office for Protection of Research Subjects at the University of California, Los Angeles as well as the UCLA Office of Animal Research Oversight. Matings were carried out overnight and females were examined for the presence of vaginal plugs, E $0.5 \mathrm{dpc}$ (days post coitum). Animal groups were of mixed gender unless otherwise stated. CD-1 wild-type mice were obtained from Charles River for bone marrow OB precursor OC precursor and calvarial bone isolation studies. B6 mice were obtained from Charles River for OVX studies. Sprague-Dawley rats were used for Nell-1 expression studies. Heterozygote carriers of the Nell-1 ${ }^{+16 \mathrm{R}}$ gene were provided by the Mammalian Genetic Research Facility at the Oak Ridge National Laboratory and were transferred with permission of the Chancellor's Animal Research Committee. The Nell-1 ${ }^{6 \mathrm{R} / 6 \mathrm{R}}$ mouse genotypes were identified from DNA extracted from clipped tails of mutant and wild-type mice. The extracted DNA was amplified using microsatellite primers D7Mit 315-L; $5^{\prime}$-TGATAACAAAACAGTCAGTA TGAAGC-3', D7Mit 315; 5'-RCTGATCCATCTGTATGATGTTACTTG-3' . All mice were housed in the light- and temperature-controlled UCLA vivarium, and provided water and feed ad libitum. Sheep were cared for at the Colorado State University according to the Veterinary Teaching Hospital institutional guidelines. All sheep were fed a grass/alfalfa mix hay and provided water and feed ad libitum. Whenever possible, animals were randomized with even distribution across treatment groups (including data presented in Figs 5 and 6).

Murine radiographic analyses. Murine samples were analysed by DXA, live microCT $/{ }^{18} \mathrm{~F}$-PET and high-resolution microCT. DXA was performed as previously described on live Nell-1 $1^{+/+}$and Nell-1 ${ }^{+/ 6 \mathrm{R}}$ littermates as follows. Scans were performed under isoflurane sedation using a Lunar PIXImus II Densiometer (GE Healthcare, Piscataway, NJ). BMD and bone mineral content (BMC) were calculated using a rectangular region of interest (ROI) encompassing the lumbar spine, or the distal left femur. Analyses were performed on 18-month-old mice; $N=12$ Nell-1 $1^{+1+}$ and 16 Nell-1 ${ }^{+/ 6 R}$ mice were used for DXA analyses. Unless otherwise stated, for all studies utilizing Nell-1 $1^{+/ 6 \mathrm{R}}$ mice, the entire colony of the studied age and gender was included for analysis. No exclusion criteria were established. Live computed tomography/positron emission tomography (CT/PET) studies were performed as follows. Fluoride ion was produced using ${ }^{18} \mathrm{O}$-water and proton bombardment using an RDS cyclotron (Siemens Preclinical Solutions). ${ }^{18} \mathrm{~F}$ Fluoride ion was produced at specific activities of $\sim 37 \mathrm{TBq} \mathrm{mmol}^{-1}$. Using an animal research committee (ARC)-approved isolated imaging chamber, all animals underwent CT/PET scanning. Mice were injected with ${ }^{18} \mathrm{~F}$-Fluroide ion via tail vein using a 27 -gauge needle. Animals were positioned in a multimodality, portable isolated bed system. Whole-body scans were performed with a 10-min acquisition time using a microPET FOCUS 200 system (Siemens Preclinical Solutions). Immediately afterwards, a noncontrast-enhanced microCT study using a microCAT II (Siemens Preclinical Solutions) imaging system was used to scan animals with a 20 -min acquisition time. PET scan images were reconstructed using filtered backprojection and an iterative three-dimensional (3D) reconstruction algorithm (maximum a posteriori). MicroCT images were created using Fledkamp reconstruction at $200-\mu \mathrm{m}$ resolution. ${ }^{18} \mathrm{~F}$-Flouride and CT data were analysed and quantified with AMIDE (A Medical Image Data Examiner). To quantify the BMD and ${ }^{18} \mathrm{~F}$ uptake, a cylindrical ROI was drawn encompassing a single lumbar vertebrae of set dimensions. Data for CT/PET images were compiled and analysed by three independent, blinded reviewers. $N=8$ mice per genotype were analysed at 1 and 6 months of age, $N=11$ Nell-1 $1^{+1+}$ mice and $12 \mathrm{Nell}-1^{+/ 6 \mathrm{R}}$ mice were analysed at 18 months of age. For PET, $N=6$ mice per genotype were analysed at 18 months of age. High-resolution, post-mortem microCT scanning and analysis were performed on neonatal whole mice $(N=10$ mice per genotype) and aged mouse spines $\left(\mathrm{N}=12 \mathrm{Nell}-1^{+/+}\right.$and $19 \mathrm{Nell}-1^{+/ 6 \mathrm{R}}$ individual vertebrae per genotype). Samples were harvested, formalin-fixed and imaged using high-resolution microCT 
(Skyscan 1172F, Skyscan, Belgium) at an image resolution of 17.8-28.2 $\mu \mathrm{m}$ and analysed using DataViewer, Recon, CTAn and CTVol softwares provided by the manufacturer. For neonatal spine CT data analysis, the ROI included the entire volume lumbar spine to quantify BMD and bone volume/tissue volume. Trabecular analysis was performed on aged mouse spine specimens, for which ROIs were drawn to include each individual lumbar vertebral body excluding the cortical bone. Reconstructions were performed using the Osirix software, using coronal cross-sectional images with a $0.25-\mu \mathrm{m}$ width. All quantitative and structural morphometric data use nomenclature described by the American Society for Bone and Mineral Research Nomenclature Committee ${ }^{58}$. MicroCT indices were compared with the published norms to ensure accuracy of analysis and reporting ${ }^{59-61}$. Whenever possible, all radiographic studies were performed and quantified in a blinded manner. Nell-1 $1^{+/+}$and Nell-1 ${ }^{+/ 6 \mathrm{R}}$ littermates look nearly identical, with the exception of a variably lighter coat colour among Nell-1 ${ }^{+/ 6 \mathrm{R}}$ mice. However, unblinding of radiographic technicians would have minimal potential for the introduction of bias, as all radiographic studies were highly routinized. ROI construction was performed in a completely blinded manner. However, a visually osteopenic skeleton among Nell-1 ${ }^{+/ 6 \mathrm{R}}$ mice in theory presented a potential for unblinding.

Nuclear magnetic resonance. NMR was used to determine total body fat mass and lean mass on live Nell- $1^{+1+}$ and Nell-1 ${ }^{+/ 6 R}$ littermates as follows. A Bruker Minispec was used with software from Echo Medical Systems (Houston, TX). In total, 12 Nell- $1^{+/+}$and 16 Nell-1 ${ }^{+/ 6 R}$ mice were used for NMR analyses. All mice were weighed before NMR analysis.

Histologic analyses. For histology, all tissues were fixed in 10\% PBS-buffered formalin. Mouse samples were decalcified in 19\% EDTA and embedded in paraffin. Five-micron-thick sections were made and stained with haematoxylin and eosin, Masson's Trichrome, Aniline Blue and TRAP staining (Sigma-Aldrich) as per the standard protocols. Analysis of Masson's Trichrome and Aniline Blue staining was performed to demonstrate the degree of mineralization between samples. Histological specimens were analysed using the Olympus BX51 microscopes and images acquired using MicroFire digital camera with the Picture Frame software (Optronics, Goleta, CA). Indirect immunohistochemistry was performed using 3-amino-9-ethylcarbazole (AEC) as chromogen, with primary antibodies listed in Supplementary Table 6. Histomorphometric analysis was performed using Adobe Photoshop. Measurements included bone area (B.Ar), percentage bone area (\% B.Ar), B.Pm, cortical width and trabecular analyses (trabecular bone width, trabecular bone number, trabecular bone spacing). Numbers of quantified images for each analysis are as follows: B.Ar $\left(N=29\right.$ Nell- $1^{+1+}$ and 26 Nell-1 $1^{+16 R}$ images), \% B.Ar $\left(N=29\right.$ Nell- $1^{+1+}$ and 26 Nell- $1^{+16 R}$ images $)$ and B.Pm $\left(N=30\right.$ Nell- $1^{+1+}$ and 29 Nell-1 $1^{+16 \mathrm{R}}$ images). Ob.N was assessed by per high-power field of Masson's Trichrome staining $\left(N=20\right.$ Nell-1 $1^{+1+}$ and 20 Nell- $1^{+16 \mathrm{R}}$ images analysed; $N=18-30$ images per treatment group analysed for Fig. 6), while Oc.N was assessed per high-power field of TRAP staining $\left(N=26\right.$ Nell-1 $1^{+1+}$ and 39 Nell-1 $1^{+/ 6 R}$ images analysed; $N=28-40$ images per treatment group analysed for Fig. 6). Cytomorphologic definition of an OB required bone-lining cells with a single round to ovoid nuclei and fairly abundant cytoplasm. Characteristic cell morphology with three or more nuclei was used to define Oc.N. Analyses of immunohistochemical staining are either reported as the number of immunoreactive cells per B.Pm, or semiquantitative measurement of relative total immunoreactivity. Semiquantitative measurements were performed using the magic wand tool in Adobe Photoshop.

Serum studies. ELISA-based serum studies were performed using serum samples as per the manufacturer's instructions. Briefly, Nell-1 $1^{+1+}$ and Nell-1 ${ }^{+/ 6 \mathrm{R}}$ littermates were bled via the retro-orbital sinus at 0900 hours on the same day after overnight fasting. Serum was collected via centrifugation and stored before examination of serum PINP, serum TRAP5b and serum CTX. All assays were obtained from ImmunoDiagnostic Systems Inc. and performed according to the manufacturer's instructions. For serum PINP, a rat/mouse enzymeimmunoassay was used $\left(N=19\right.$ and 17 mice for Nell-1 $1^{+1+}$ and Nell- $1^{+/ 6 \mathrm{R}}$ mice, respectively). For serum CTX, a RatLaps Enzymeimmunoassay was used ( $N=12$ and 22 mice for Nell- $1^{+1+}$ and Nell-1 $1^{+76 R}$ mice, respectively). For serum TRAP5b, a MouseTRAP solid-phase immunofixed enzyme activity assay was used $(N=10$ and 9 mice for Nell-1 $1^{+1+}$ and Nell-1 ${ }^{+16 \mathrm{R}}$ mice, respectively; kits listed in Supplementary Table 7). Gene expression was performed after microdissection of lower lumbar vertebral bodies, following by mRNA extraction and quantitative real-time (RT)-PCR. $N=3$ samples (Fig. 1c) or 4 samples (Fig. 3c) per genotype were used for analysis. Primers are listed in Supplementary Table 8.

Biomechanical analyses. For biomechanical testing, two methods were independently used: the BioDent Reference Point Indenter and computerized simulation (finite element analysis, FEA). Analysis of the S1 vertebral body was performed on all mice. Lumbar vertebrae could not be used, as the spines in the Nell-1 $1^{+/ 6 \mathrm{R}}$ mice were too fragile for analysis. The indenter was placed on the dorsal periosteal surface for measurement acquisition. The following settings were used: $2 \mathrm{~N}$ indentation force, 2 indentations per second and 10 indentations per measurement. Total indentation distance was calculated by measuring the maximum indentation distance achieved during a measurement. Indentation distance increase (IDI) was calculated by measuring the difference between the depths reached at peak force during the first indentation cycle and last indentation cycle. Unloading stiffness is calculated by evaluating the top portion of the unloading section of the force displacement curve. $N=3$ mice per genotype, with $N=6$ measurements per mouse used for indentation studies.

FEA (biomechanical) was performed using microcT images converted to DICOM files using the SKyScan Dicom Converter software (DicomCT application, Skyscan 1172F, Skyscan). Tetrahedral 3D mesh models were created using an VOI of either the lumbar spine (level 4) using the ScanIP software (Simpleware Limited). A constant thickness of $0.54 \mathrm{~mm}$ was used for both VOIs. FEAs were performed using the ABAQUS software (Dassault Systèmes) with boundary conditions set as encastre, constrained in all directions. Next, we applied a uniform compressive pressure of $0.5 \mathrm{MPa}$ on the superior surface of the Volume of interest (VOI). The von Mises stress experienced and total strain energy of the samples were analysed. $N=8$ mice per genotype for FEA analyses.

Dynamic histomorphometric analyses. For bone fluorescent-labelling studies, mice were injected intraperitoneally with calcein $\left(20 \mathrm{mg} \mathrm{kg}^{-1}\right)$ and alizarin red complexon $\left(50 \mathrm{mg} \mathrm{kg}^{-1}\right)$ at 9 and 2 days before being killed, respectively. Lumbar vertebrae were dissected, fixed in $70 \%$ ethanol, dehydrated and embedded undecalcified in methyl methacrylate. Coronal sections at 5- $\mu \mathrm{m}$ thickness were analysed using the OsteoMeasure morphometry system (Osteometrics, Atlanta, GA, USA). For dynamic histomorphometry, the mineral apposition rate $(\mu \mathrm{m}$ per day), the distance between the midpoints of the two labels divided by the time between the midpoints of the interval were measured in unstained sections under ultraviolet light and used to calculate the bone formation rate with a bone surface referent (BFR/BS, $\mu \mathrm{m}^{3} \mu \mathrm{m}^{-2}$ per year). The bone formation rate per bone surface $(\mathrm{BFR} / \mathrm{BS})$ is the volume of mineralized bone formed per unit time and per unit bone surface. The fixed area and location at $\times 200$ magnification of lumbar vertebral 4-5 (L4-5) was selected as the ROI $(N=3$ mice and 6 measurement fields per treatment group). All image acquisition and analyses were performed in a blinded manner.

OB precursor culture and experiments. Mouse $\mathrm{OB}$ precursors were harvested by flushing the femoral marrow cavities and harvesting adherent cells on standard culture-treated plates. For isolation of Nell-1 $1^{+1+}$ and Nell-1 $1^{+/ 6 \mathrm{R}} \mathrm{OB}$ precursors, cells were isolated from 18-month-old littermates ( $N=3$ mice per genotype). For additional experiments using wild-type $\mathrm{OB}$ precursors, 3-month-old $\mathrm{CD}-1$ animals were used. Proliferation and osteogenic differentiation and assessments were performed with or without recombinant human (rh)NELL-1 protein $\left(100-300 \mathrm{ng} \mathrm{ml}^{-1}\right)$. The osteogenic differentiation medium was constituted with $10 \mathrm{mM} \beta$-glycerophosphate and $50 \mu \mathrm{M}$ ascorbic acid (Fisher Scientific, Pittsburgh, $\mathrm{PA}$ ) in high-glucose DMEM, 10\% fetal bovine serum (FBS), 1\% penicillin/ streptomycin (GIBCO, Invitrogen, Carlsbad, CA). To assess early to intermediate OB differentiation, ALP staining and quantification was performed in each case normalized to total protein content in sister wells. To assess bone nodule formation, Alizarin red staining and quantification was performed using CPC leaching and photometric quantification, normalized to total protein content. Quantitative real-time PCR was performed in triplicate wells per RNA isolate. Primers are listed in Supplementary Table 8. All experiments were performed in triplicate wells. Passage 2 OB precursors only were used for all assays. For al in vitro studies, technical execution of the experiments was performed in an unblinded manner, unless otherwise noted.

OC precursor resorption experiments. Calvarial discs were obtained using a 2-mm punch biopsy from the mid-parietal bone of postnatal day 7, CD-1, wild-type mice. Discs were processed by gentle removal of periosteal and dural tissues and stored in $100 \%$ ethanol to decellularize the tissues at $4{ }^{\circ} \mathrm{C}$. Mouse OC precursors were obtained by femoral and tibial marrow flushing. For isolation of Nell-1 $1^{+1+}$ and Nell-1 ${ }^{+16 \mathrm{R}}$, OC precursors were isolated from 18-month-old littermates $(N=3$ mice per genotype used). For additional experiments using wild-type OC precursors, 3-month-old CD-1 animals were used. The product of total bone marrow flush was cultured in $25 \mathrm{ng} \mathrm{ml}^{-1}$ recombinant mouse M-CSF (R\&D Systems, Minneapolis, MN) overnight. The nonadherent cells were then cultured on calvarial discs for resorption assays in phenol red-free $\alpha \mathrm{MEM}$ media $10 \% \mathrm{FBS}, 25 \mathrm{ng} \mathrm{ml}^{-1}$ recombinant mouse Macrophage Colony Stimulating Factor (rmM-CSF) and $100 \mathrm{ng} \mathrm{ml}^{-1}$ recombinant soluble RANKL (R\&D Systems), with or without rhNELL-1 $\left(0-1200 \mathrm{ng} \mathrm{ml}^{-1}\right)$. After 5 days, calvarial discs were harvested, washed in $1 \times \mathrm{PBS}$ and stained with $1 \%$ Toluidine Blue dye in dilute Sodium Borate (Fisher Scientific). Calvarial discs were photographed and quantification of intensity blue staining (indicating the presence of bone) was performed using the magic wand tool in Adobe Photoshop CS5 $(N=9$ and 11 samples for Nell- $1^{+1+}$ and Nell-1 ${ }^{+16 \mathrm{R}}$ samples, respectively). Next, scanning electron microscopy was performed as using a Nova 230 microscope (FEI, Hillsboro, Oregon) in a low vacuum mode operated at 40 Pascal. Accelerating voltage was used at $10 \mathrm{kV}$ with a working difference varying between 4.8 and $5.7 \mathrm{~mm}$. Samples were analysed at a magnification of $\times 200$. The MeX 3D software version 5.1 (Alicona Imaging 
Corporation, Austria) was used to analyse the mean resorption pit depth and the mean roughness of the calvarial discs. In order to measure the depth of the pits, several lines were drawn through each suspected pit at $30^{\circ}$ intervals; the line that provided the largest difference between peak and valley was used, determining the distance in $\mathrm{mm}$ between the peak and valley measurements. Resorption pits were defined as having a minimum depth of $1.5 \mu \mathrm{m}(N=9$ measurements per genotype). Average roughness was measured by drawing six lines across each sample at 100$\mu \mathrm{m}$ intervals using the MeX software $\left(N=36\right.$ and 29 measurements for Nell-1 $1^{+1+}$ and Nell-1 $1^{+/ 6 \mathrm{R}}$ samples, respectively). The calculated roughness of each line was then used to calculate the mean roughness per treatment group. s.e.m. measurements were performed in a blinded manner.

M2-10B4 and RAW264.7 cell culture. The M2-10B4 cell line, a clone derived from BMSCs from a (C57BL/6J X C3H/HeJ) F1 mouse, was purchased from American Type Culture Collection (ATCC no. CRL-1972, Lot no. 58696031, Manassas, VA) and used for experiments within 6 months of mycoplasma contamination testing. RAW264.7 cells were a kind gift from the laboratory of Dr Tintut. Cells were maintained in growth medium (RPMI 1640 supplemented with $10 \% \mathrm{FBS}, 1 \mathrm{mM}$ sodium pyruvate and $100 \mathrm{U} \mathrm{ml}^{-1}$ penicillin/streptomycin). RAW264.7 cells were cultured in $\alpha \mathrm{MEM}+10 \% \mathrm{FBS}$. Wnt/ $\beta$-catenin signalling was assayed using four methods: immunocytochemistry for active $\beta$-catenin, western blotting for nuclear $\beta$-catenin, TCF/LEF1 reporter activity (TOPFLASH) and qRT-PCR. Experiments used recombinant WNT3A as a positive control. For immunocytochemistry, M2-10B4 cells were seeded on Millicell EX Slides (PEZGS0816, Millipore) at $5 \times 10^{4}$ cells per well in RPMI $1640+10 \%$ FBS for $24 \mathrm{~h}$ and serum-starved in RPMI $1640+1 \%$ FBS overnight. After 2-h treatment, cells were fixed using ice-cold acetone for $10 \mathrm{~min}$. Anti-active $\beta$-catenin antibody (Millipore) was applied at a dilution of 1:200. The ABC complex (Vector Laboratories, Burlingame, CA) was applied to the sections following incubation with biotinylated secondary antibody (Dako). AEC substrate (Dako) was used as a chromogen, and the sections were lightly counterstained with haematoxylin. Photomicrographs were acquired using Olympus BX51 ( $\times 200$ magnification lens, UPLanFL, Olympus; $N=4$ wells per treatment group). For western blot analysis, nuclear and cytoplasmic protein was isolated using the NE-PER Nuclear and Cytoplasmic Extraction Kit (Thermo Scientific, Rockford, IL). Western blot analysis was performed using an antibody against $\beta$-catenin at a dilution of 1:1,000 ( $N=3$ wells per treatment group). For TCF/LEF1 reporter activity, cells were transfected with $20 \mu \mathrm{g}$ Super $(16 \times)$ TOPFLASH (TCF/LEF1 Reporter Plasmid) and $1 \mu \mathrm{g}$ Renilla Luciferase plasmids for $24 \mathrm{~h}$ and then seeded at $4.0 \times 10^{3}$ cells per well in 96-well plates. Cells were starved in RPMI $1640+1 \%$ FBS overnight and then treated with rhNELL-1. Luciferase activity was measured $48 \mathrm{~h}$ after treatment using the Dual Luciferase Reporter Assay System (Promega, Madison, WI) as per the manufacturer's instructions ( $N=4$ wells per treatment group).

Lentiviral vector for runx2 reporter assay. The transduction plasmid for preparing lentiviral vector carrying the Runx2 P1-EGFP expression cassette (Runx2-EGFP reporter) was prepared by substituting the CMV promoter in the pRRL-CPPT-CMV-X-PRE-SIN plasmid with the mouse Runx2 P1 promoter. The mouse Runx2 P1 promoter was obtained with PCR of mouse genomic DNA (forward primer: $5^{\prime}$-gcgaattactcgagagcagcactgttgctcagaa- $3^{\prime}$; reverse primer: $5^{\prime}$-gcgaatgcccgggtcacacaatccaaaaaagc- $\left.3^{\prime}\right)$. 293T cells were co-transfected with the transduction plasmids, the package plasmid pCMV-dR8.2-vprX and the envelope plasmid pCMV-VSVG. The viral vectors were collected at 2-4 days post transfection, filtered and concentrated, and the concentrations of viral vector were quantified by counting the core protein p24 using ELISA assay. For cell transfection, M2-10B4 cells were seeded in 24 -well cell culture plates at $2 \times 10^{4}$ cells per well $16 \mathrm{~h}$ before infection. Viral vectors with p24 counts of $0.4 \mu \mathrm{g}$ were added to each well in 24-well plates. Three hours post infection, the viral vectors were washed away and fresh medium was added to the cultures. At $24 \mathrm{~h}$ post infection, rhNELL-1 was added into the culture media. Cells were trypsinized and collected 3 days post infection. Flow cytometry was performed to quantify green fluorescent protein (GFP) expression in the collected cells using a Cytomics FC500 cell sorter (Beckman Coulter, Brea, CA). Cells infected with mock vector were used as the negative control to establish gates. The percentages of GFP-positive cells were counted to quantify the expression of GFP in the infected cultures $(N=4$ wells per treatment). For select experiments, DKK-1 $\left(100 \mathrm{ng} \mathrm{ml}^{-1}\right)$ or XAV939 $(1 \mu \mathrm{M})$ were used ( $N=4$ wells per treatment group).

siRNA experiments. RNA knockdown experiments were performed in M2-10B4 and RAW264.7 cells using chemically synthesized and annealed siRNA specific to integrin $\beta 1$ (Santa Cruz, sc-35675), with $N=3$ wells per treatment group. Integrin $\beta 1$ siRNA is a pool of four different siRNA duplexes: (A) Sense: $5^{\prime}$-GAACGGA UUUGAUGAAUGAtt-3', Antisense: $5^{\prime}$-UCAUUCAUCAAAUCCGUUCtt- $3^{\prime}$, (B) Sense: $5^{\prime}$-CAUUGGCUUUGGCUCAUUUtt- $3^{\prime}$, Antisense: $5^{\prime}$-AAAUGAGCCA AAGCCAAUGtt-3', (C) Sense: $5^{\prime}$-CAACCUGUUUACAAGGAAUtt- $3^{\prime}$, Antisense: $5^{\prime}$-AUUCCUUGUAAACAGGUUGtt-3', (D) Sense: 5'-UUUGGACACUGGUCCA UGUtt- $3^{\prime}$, Antisense: $5^{\prime}$-ACAUGGACCAGUGUCCAAAtt- $3^{\prime}$. Control siRNA was used (SC-37007) with sequence r(5'-UUCUCCGAACGUGUCACGU)d(TT)- $3^{\prime}$. When cells reached $30 \%$ confluence, cells were transfected with $50 \mathrm{nM}$ integrin $\beta 1$
siRNA or nontarget negative control siRNA (Santa Cruz) using Lipofectamine RNAiMax (Invitrogen). Efficiency of knockdown was validated using western blot analysis.

Human BMSC culture. Human BMSCs were obtained from $N=5$ patients, with or without a history of osteoporosis (see Supplementary Table 2 for a description of patient samples). Samples were obtained during a surgical procedure, predominantly for fracture or joint replacement surgery (see Supplementary Table 2), and with UCLA IRB exemption (IRB no. 11-000724). Samples were de-identified to protect the privacy of the patient. As no direct contact and no risk was poised to the patient, neither informed consent nor authorization was obtained, as per the University guidelines. Both nonosteoporotic and osteoporotic patients were without major medical comorbidities. Bone marrow tissues with bone chips were obtained and stored on ice during transport. These tissues were next digested with Type II collagenase $\left(2 \mathrm{mg} \mathrm{ml}^{-1}\right)$ under agitation and followed by Ficoll centrifugation. The mononuclear cells were separated and seeded into T-75 flasks for cell expansion. hBMSCs were obtained as adherent cells on standard culture-treated plates. Cells were expanded in $\alpha \mathrm{MEM}+20 \% \mathrm{FBS}$ on 10 -mm cell culture plates. All experiments were performed in triplicate for each patient sample To manipulate Nell-1 signalling, hBMSCs were transduced with Ad-Nell-1 or Ad-GFP at multiplicity of infection 50 pfu per cell. First, cells were either seeded for osteogenic differentiation assays $\left(2 \times 10^{4}\right.$ cells per well $)$ in 24 -well plates. Osteogenic differentiation medium consisted of $50 \mu \mathrm{g} \mathrm{ml}^{-1}$ ascorbic acid and $10 \mathrm{mM} \beta$-glycerophosphate. Staining for AR was performed at 11 days of differentiation. Next, Wnt/ $\beta$-catenin signalling activity was assayed on the gene and protein level. mRNA analysis was performed 3 days after transduction. For western blot analysis, cell lysate was collected 3 days after transduction. Nuclear and cytoplasmic fractions were extracted using the NE-PER Nuclear and Cytoplasmic Extraction Kit (Thermo Scientific). Western blot analysis was performed with anti- $\beta$-catenin (610153, BD Biosciences), anti- $\beta$-actin (sc-1616, Santa Cruz) and anti-H3 (AB8898, Abcam). Please see Supplementary Table 6 for specific antibody concentrations used. Quantification was performed using Image Pro. Taqman primers were used for human NELL-1 (Hs00196243_m1) and human GAPDH (Hs99999905_ml). All experiments using human BMSC were performed in triplicate wells.

Assessments of NELL-1/integrin $\beta 1$ interaction. Confirmation of NELL-1 binding to integrin $\beta 1$ was performed in two methods, including thermal shift assays and computational modelling. The thermal shift assay was performed using the 7,300 Real Time PCR System (Applied Biosystems, CA). Before use, the environmentally sensitive fluorescent dye SYPRO Orange stock solution in dimethylsulphoxide $(5,000 \times$, Sigma) was diluted 1:125 in PBS. The samples were prepared in a 96-well plate in triplicate containing $2 \mu \mathrm{g}$ NELL-1 and/or $1 \mu \mathrm{g}$ integrin $\alpha 3 \beta 1$ (R\&D Systems, 2840-A3-050) and freshly diluted SYPRO orange in PBS buffer $(0.01 \mathrm{M}, \mathrm{pH} 7.4)$ with the total volume of $15 \mu \mathrm{l}$, and then the plate was sealed with optical quality sealing tape and centrifuged at 4,000 r.p.m. for $2 \mathrm{~min}$. The fluorescent intensity was monitored as the plate was heated from 25 to $89^{\circ} \mathrm{C}$ in an increment of $1^{\circ} \mathrm{C} \mathrm{min}^{-1}$. Second, computational modelling was performed between NELL-1 and the extracellular domain of integrin $\beta 1$ based on RosettaDock. The three top-scoring models of NELL-1 to integrin $\beta 1$ predicted by docking simulation are depicted in Supplementary Fig. 4 .

TOPGAL mouse intrafemoral Ad-Nell-1 injection. Wnt reporter TOPGAL mice were injected by intrafemoral injection with Ad-Nell-1 $\left(5 \times 10^{12} \mathrm{pfu} \mathrm{ml}^{-1}\right)$ or Ad-CMV-Null control (Vector BioLabs, Philadelphia, PA). Mice were randomized to each treatment group. Block randomization was used to ensure equal sample sizes. A percutaneous, infrapatellar approach was used, with a 26-gauge needle, and a $10-\mu l$ total injection volume. Animals were killed 1 and 2 weeks post injection for the analysis of $\beta$-galactosidase expression within the marrow space. For histological analysis, whole-mount X-gal staining was performed 1 week post injection followed by paraffin embedding and examination of serial sections. $N=3$ mice per treatment group. For quantification, flow cytometry was performed 2 weeks post injection. Bone marrow cells were flushed from the femur. Next, the femoral shaft was finely minced and the bone chips were digested with collagenase II $\left(3 \mathrm{mg} \mathrm{ml}^{-1}\right)$ for $2 \mathrm{~h}$. The resultant cells from both bone marrow flush and cell digestion were combined, pelleted and washed twice with PBS $+1 \%$ FBS $\beta$-galactosidase substrate FDG (Invitrogen F1179) was then added to cells under hypotonic shock for $1 \mathrm{~min}$ and run for flow cytometry (LSRII, BD Bioscience). $N=4$ mice per treatment group.

Mouse OVX and rhNELL-1 intravenous injection. OVX was performed on B6 mice with age-matched Sham controls at 12 weeks of age, using a 5-mm dorsal incision. Anaesthesia was performed with Isoflurane (3-5\% for induction and $1.5-2 \%$ for maintenance). Pre-operative analgesia (Buprenorphine) was given once before surgery and twice daily for 2 days postoperatively. Five weeks post OVX, rhNELL-1 delivery by intravenous injection was instituted, by lateral tail vein injection using a 25 -gauge needle. Animals were assigned to treatment groups by simple randomization. Drug administrators were completely blinded as to the treatment groups. The dosage of rhNELL-1 $\left(1.25 \mathrm{mg} \mathrm{kg}^{-1}\right)$ was obtained from 
pilot studies examining a wide range of doses. PBS served as vehicle control. Each injection consisted of a total $0.1 \mathrm{ml}$ solution, and the injection was given every $48 \mathrm{~h}$ for the study period. Analyses were as per above, including DXA, live CT/PET, high-resolution microCT and quantification, histology and immunohistochemical staining. Animal numbers for each analysis were as follows (Sham Control, Sham rhNELL-1, OVX Control, OVX rhNELL-1): DXA (6, 7, 6 and 9 mice), high-resolution microCT $(5,5,6,9$ mice), Osteocalcin quantification $(18,23,25$ and 30 images), TRAP quantification (28, 34, 28 and 40 images) and FEA $(N=6,7$, 6,9 mice). ROIs for analysis included the lumbar vertebrae. MicroCT indices were compared with the published norms to ensure the accuracy of analysis and reporting ${ }^{59-61}$. In addition, post-mortem analysis of uterus weight was obtained to confirm OVX. In select experiments, fluorescein isothiocyanate conjugation was employed to determine the pharmacokinetics of rhNELL-1 systemic delivery.

Sheep experiment preparation and surgery. Osteoporosis was induced through OVX, controlled diet and steroid induction in eight adult ewes. Post OVX, three intramuscular injections of $500 \mathrm{mg}$ methylprednisolone acetate (Depomedrol) were administered at 3-week intervals starting 2 weeks post operation. Special lowcalcium and low-vitamin D osteoporosis diets were formulated in cooperation with Purina LabDiet and were fed to the sheep for 8 months post OVX. On the basis of pre-established inclusion criteria, of eight sheep in total, the six sheep with highest response to osteoporotic induction were used for further study. Successful induction of osteoporosis was confirmed using DXA imaging and quantification. The contents of material for sheep intrabody vertebral injection are in Supplementary Table 3 , and included hyaluronic acid, $\beta$-tricalcium phosphate, with or without rhNELL-1 protein. Block randomization was used to ensure equal treatment group sizes. Surgeons were completely blinded to the treatment group. As injection contents appeared visually identical between groups, no unblinding of the operating surgeons was observed. Surgery was performed 4 months post OVX on $N=6$ osteoporotic sheep. A ventrolateral incision was made for a retroperitoneal approach. Induction of anaesthesia was performed by Valium or Midazolam or propofol along with ketamine through a venous catheter placed in an ear vein. Following induction, animals were intubated and transferred to Isoflurane in oxygen inhalant titrated to maintain a plane of surgical anaesthesia. Dissection was carried out to expose the cranial aspect of the L1-L5 vertebral bodies. A 3.2-mm unicortical drill hole was created on the left lateral aspect of the vertebral body. An introducer with stylet that perfectly fitted the drillhole was then inserted, ensuring an airtight, watertight seal. The stylet was then removed, and using a luer lock syringe the implant material injected into the introducer, and the stylet reinserted so as to push all materials into the cancellous bone of the vertebral body. No leakage of injection contents was observed. Bone wax was used to seal the drill hole. Three different vertebral levels were instrumented per sheep (L1, L3 and L5). Three sheep were injected with the control vehicle and three sheep were injected with the treatment. Thus, final group numbers included $N=9$ control-treated vertebrae and $N=3$ vertebrae per treatment dose. Postoperative analgesia consisted of phenylbutazone for 3 days postoperatively, with close indoor monitoring for 2 weeks postoperatively.

Sheep experiment analyses. Analysis was performed using DXA, CT and microCT, FEA, histology and histomorphometry. DXA scans were performed pre- and post-induction (Konica Minolta mc5430DL). Next, a Philip's GEMINI TF Big Bore CT machine (0.8 $\mathrm{mm}$ thickness) was used to analyse bone formation postoperatively, monthly until being killed at 3 months. Although serial live animal imaging was performed, data evaluation was performed at the study end point (no interim evaluation of the results performed). Post-mortem, high-resolution microCT scanning of individual vertebrae was performed as per mouse studies. Cortical and trabecular analyses were performed on post-mortem individual sheep vertebrae. Cortical measurements were obtained per vertebral body either assessing the surface of injection or the opposing cortical surface. Serial measurements along the cortex were obtained $(N=162$ control-treated measurements and $N=55$ measurements per treatment dose). Trabecular measurements were made using an ROI excluding the cortical bone. FEA (biomechanical) was performed using microcT images converted to DICOM files using the SKyScan Dicom Converter software (DicomCT application, Skyscan 1172F, Skyscan). Tetrahedral 3D mesh models were created by drawing a rectangular VOI directly underneath the injection tract, using the ScanIP software (Simpleware Limited). FEAs were performed using the ABAQUS software (Dassault Systèmes) with boundary conditions set as encastre, constrained in all directions. Next, we applied a uniform compressive pressure of $0.5 \mathrm{MPa}$ on the superior surface of the spine, to reproduce human intradiscal pressure experienced in relaxed standing. The von Mises stress experienced and total strain energy of the samples were analysed. All radiographic analyses, including ROI construction, were performed in a blinded manner. The significant and visually observed anabolic effect of rhNELL-1 in theory presented a potential for unblinding. For histology, tissues were formalin-fixed, resinembedded and stained with haematoxylin and eosin, Goldner's Modified Trichrome and Von Kossa-MacNeal's Tetrachrome. Histological specimens were analysed using the Olympus BX51 microscopes and images acquired using MicroFire digital camera with the Picture Frame software (Optronics, Goleta, CA). Histomorphometry was performed as per mouse studies. Ob.N was assessed by $\times 400$ images of Von Kossa-MacNeal's Tetrachrome $(N=10,9,9$ and 9 images per treatment group, respectively), while Oc.N was assessed by $\times 200$ images of Goldner's Modified Trichrome staining $(N=9,10,9$ and 9 images per treatment group, respectively). Characteristic cell morphology with three or more nuclei was used to define Oc.N.

Statistical analysis. Quantitative data are expressed at mean \pm s.e.m. unless otherwise described, with ${ }^{\star} P<0.05$ and ${ }^{*} P<0.01$ considered significant. A Shapiro-Wilk test for normality was performed on all data sets. Homogeneity was confirmed by a comparison of variance test. Parametric data were analysed using an appropriate Student's $t$-test when two groups were being compared, or a one-way analysis of variance was used when more than two groups were compared, followed by a post hoc Tukey's test to compare the two groups. Nonparametric data were analysed with a Mann-Whitney $U$-test when two groups were being compared or a Kruskal-Wallis one-way analysis when more than two groups were compared. As appropriate, adjustments for multiple comparisons were performed using a Bonferroni correction (see Supplementary Tables 9 and 10). Sample size calculations were performed for experiments presented in Figs 5 and 6 and Supplementary Figs 6-8 as follows: for experiments presented in Fig. 5 and Supplementary Fig. 6 initial animal numbers were on the basis of an $\alpha=0.05$, power $=0.8$ and an anticipated effect size of 3.73 (on the basis of our previously published data in sheep spinal fusion $)^{27}$. For experiments presented in Fig. 6 and Supplementary Figs 7 and 8, initial animal numbers were on the basis of an $\alpha=0.05$, power $=0.8$ and an anticipated effect size of 2.69 (on the basis of our previously published data in local rhNELL-1 injection in ovariectomized rats) ${ }^{62}$. No sample size calculations were performed for animal studies in Fig. 1, Supplementary Figs 1 and 2, as all available transgenic animals were examined.

\section{References}

1. Becker, D. J., Kilgore, M. L. \& Morrisey, M. A. The societal burden of osteoporosis. Curr. Rheumatol. Rep. 12, 186-191 (2010).

2. Rasmussen, H. \& Bordier, P. The cellular basis of metabolic bone disease. N. Engl. J. Med. 289, 25-32 (1973).

3. Mellis, D. J., Itzstein, C., Helfrich, M. H. \& Crockett, J. C. The skeleton: a multifunctional complex organ: the role of key signalling pathways in osteoclast differentiation and in bone resorption. J. Endocrinol. 211, 131-143 (2011).

4. Canalis, E., Giustina, A. \& Bilezikian, J. P. Mechanisms of anabolic therapies for osteoporosis. N. Engl. J. Med. 357, 905-916 (2007).

5. Martin, T., Gooi, J. H. \& Sims, N. A. Molecular mechanisms in coupling of bone formation to resorption. Crit. Rev. Eukaryot. Gene Expr. 19, 73-88 (2009).

6. Sims, N. A. \& Gooi, J. H. Bone remodeling: multiple cellular interactions required for coupling of bone formation and resorption. Semin. Cell Dev. Biol. 19, 444-451 (2008).

7. Zara, J. et al. High doses of BMP2 induce structurally abnormal bone and inflammation in vivo. Tissue Eng. A 17, 1389-1399 (2011).

8. Jensen, E. D. et al. Bone morphogenic protein 2 directly enhances differentiation of murine osteoclast precursors. J. Cell Biochem. 109, 672-682 (2010).

9. Cowan, C. M. et al. Bone morphogenetic protein 2 and retinoic acid accelerate in vivo bone formation, osteoclast recruitment, and bone turnover. Tissue Eng. 11, 645-658 (2005)

10. Cajanek, L., Adlerz, L., Bryja, V. \& Arenas, E. WNT unrelated activities in commercially available preparations of recombinant WNT3a. J. Cell Biochem. 111, 1077-1079 (2010).

11. Jing, L., Lefebvre, J. L., Gordon, L. R. \& Granato, M. Wnt signals organize synaptic prepattern and axon guidance through the zebrafish unplugged/MuSK receptor. Neuron 61, 721-733 (2009).

12. Willert, K. et al. Wnt proteins are lipid-modified and can act as stem cell growth factors. Nature 423, 448-452 (2003).

13. Wagner, E. R. et al. The therapeutic potential of the Wnt signaling pathway in bone disorders. Curr. Mol. Pharmacol. 4, 14-25 (2011).

14. Minear, S. et al. Wnt proteins promote bone regeneration. Sci. Transl. Med. 2, 29 ra30 (2010).

15. Li, X. et al. Inhibition of sclerostin by monoclonal antibody increases bone formation, bone mass, and bone strength in aged male rats. J. Bone Miner. Res. 25, 2647-2656 (2010).

16. Tian, X. et al. Treatment with a sclerostin antibody increases cancellous bone formation and bone mass regardless of marrow composition in adult female rats. Bone 47, 529-533 (2010).

17. Ominsky, M. S. et al. Two doses of sclerostin antibody in cynomolgus monkeys increases bone formation, bone mineral density, and bone strength. J. Bone Miner. Res. 25, 948-959 (2010).

18. Padhi, D., Jang, G., Stouch, B., Fang, L. \& Posvar, E. Single-dose, placebocontrolled, randomized study of AMG 785, a sclerostin monoclonal antibody J. Bone Miner. Res. 26, 19-26 (2011).

19. Ting, K. et al. Human NELL-1 expressed in unilateral coronal synostosis J. Bone Miner. Res. 14, 80-89 (1999).

20. Zhang, X. et al. Craniosynostosis in transgenic mice overexpressing Nell-1. J. Clin. Invest. 110, 861-870 (2002). 
21. Desai, J. et al. Nell1-deficient mice have reduced expression of extracellular matrix proteins causing cranial and vertebral defects. Hum. Mol. Genet. 15, 1329-1341 (2006).

22. Shen, J. et al. NELL-1 promotes cell adhesion and differentiation via Integrinbeta1. J. Cell. Biochem. 113, 3620-3628 (2012).

23. Zhang, X. et al. Nell-1, a key functional mediator of Runx2, partially rescues calvarial defects in Runx2(+/-) mice. J. Bone Miner. Res. 26, 777-791 (2011).

24. Aghaloo, T. et al. Nell-1-induced bone regeneration in calvarial defects. Am. J. Pathol. 169, 903-915 (2006).

25. Li, W. et al. Delivery of lyophilized Nell-1 in a rat spinal fusion model. Tissue Eng. A 16, 2861-2870 (2010).

26. Li, W. et al. Nell-1 enhances bone regeneration in a rat critical-sized femoral segmental defect model. Plastic Reconstruct. Surg. 127, 580-587 (2011).

27. Lu, S. S. et al. The osteoinductive properties of Nell-1 in a rat spinal fusion model. Spine J. 7, 50-60 (2007).

28. Karasik, D. et al. Genome-wide pleiotropy of osteoporosis-related phenotypes: the Framingham Study. J. Bone Miner. Res. 25, 1555-1563 (2010).

29. Krishnan, V., Bryant, H. U. \& Macdougald, O. A. Regulation of bone mass by Wnt signaling. J. Clin. Invest. 116, 1202-1209 (2006).

30. James, A. W. et al. A new function of Nell-1 protein in repressing adipogenic differentiation. Biochem. Biophys. Res. Commun. 411, 126-131 (2011).

31. Hasebe, A. et al. The C-terminal region of NELL1 mediates osteoblastic cell adhesion through integrin alpha3beta1. FEBS Lett. 586, 2500-2506 (2012).

32. Legate, K. R., Wickstrom, S. A. \& Fassler, R. Genetic and cell biological analysis of integrin outside-in signaling. Genes Dev. 23, 397-418 (2009).

33. Rallis, C., Pinchin, S. M. \& Ish-Horowicz, D. Cell-autonomous integrin control of Wnt and Notch signalling during somitogenesis. Development 137, 3591-3601 (2010).

34. Gibson, G. J., Francki, K. T., Hopwood, J. J. \& Foster, B. K. Human and sheep growth-plate cartilage type $\mathrm{X}$ collagen synthesis and the influence of tissue storage. Biochem. J. 277, 513-520 (1991).

35. Wilke, H. J., Kettler, A., Wenger, K. H. \& Claes, L. E. Anatomy of the sheep spine and its comparison to the human spine. Anat. Rec. 247, 542-555 (1997).

36. Rehman, I., Smith, R., Hench, L. L. \& Bonfield, W. Structural evaluation of human and sheep bone and comparison with synthetic hydroxyapatite by FT-Raman spectroscopy. J. Biomed. Mater. Res. 29, 1287-1294 (1995).

37. Aerssens, J., Boonen, S., Lowet, G. \& Dequeker, J. Interspecies differences in bone composition, density, and quality: potential implications for in vivo bone research. Endocrinology 139, 663-670 (1998).

38. Spencer, G. J., Utting, J. C., Etheridge, S. L. \& Arnett, T. R. Genever PG. Wnt signalling in osteoblasts regulates expression of the receptor activator of NFkappaB ligand and inhibits osteoclastogenesis in vitro. J. Cell Sci. 119, 1283-1296 (2006).

39. Qiang, Y. W. et al. Characterization of Wnt/beta-catenin signalling in osteoclasts in multiple myeloma. Br. J. Haematol. 148, 726-738 (2010).

40. Glass, 2nd D. A. et al. Canonical Wnt signaling in differentiated osteoblasts controls osteoclast differentiation. Dev. Cell 8, 751-764 (2005).

41. Baron, R. \& Rawadi, G. Targeting the Wnt/beta-catenin pathway to regulate bone formation in the adult skeleton. Endocrinology 148, 2635-2643 (2007).

42. Albers, J. et al. Canonical Wnt signaling inhibits osteoclastogenesis independent of osteoprotegerin. J. Cell Biol. 200, 537-549 (2013).

43. Gronthos, S., Simmons, P. J., Graves, S. E. \& Robey, P. G. Integrin-mediated interactions between human bone marrow stromal precursor cells and the extracellular matrix. Bone 28, 174-181 (2001).

44. Bikle, D. D. Integrins, insulin like growth factors, and the skeletal response to load. Osteoporos. Int. 19, 1237-1246 (2008).

45. Duong, L. T., Lakkakorpi, P., Nakamura, I. \& Rodan, G. A. Integrins and signaling in osteoclast function. Matrix Biol. 19, 97-105 (2000).

46. Schmidt, S. et al. Kindlin-3-mediated signaling from multiple integrin classes is required for osteoclast-mediated bone resorption. J. Cell Biol. 192, 883-897 (2011).

47. Rodriguez, J. P., Astudillo, P., Rios, S. \& Pino, A. M. Involvement of adipogenic potential of human bone marrow mesenchymal stem cells (MSCs) in osteoporosis. Curr. Stem Cell Res. Ther. 3, 208-218 (2008).

48. Fernando, T. L., Kim, S. S. \& Mohler, D. G. Complete pelvic ring failure after posterior iliac bone graft harvesting. Spine 24, 2101-2104 (1999).

49. Balseiro, S. \& Nottmeier, E. W. Vertebral osteolysis originating from subchondral cyst end plate defects in transforaminal lumbar interbody fusion using rhBMP-2. Report of two cases. Spine J. 10, e6-e10 (2010).

50. Kaneko, H. et al. Direct stimulation of osteoclastic bone resorption by bone morphogenetic protein (BMP)-2 and expression of BMP receptors in mature osteoclasts. Bone 27, 479-486 (2000).

51. Khan, S. N. \& Lane, J. M. The use of recombinant human bone morphogenetic protein-2 (rhBMP-2) in orthopaedic applications. Expert Opin. Biol. Ther. 4, 741-748 (2004)

52. Jin, Z. et al. Hypermethylation of the nel-like 1 gene is a common and early event and is associated with poor prognosis in early-stage esophageal adenocarcinoma. Oncogene 26, 6332-6340 (2007).
53. Mori, Y. et al. A genome-wide search identifies epigenetic silencing of somatostatin, tachykinin-1, and 5 other genes in colon cancer. Gastroenterology 131, 797-808 (2006).

54. Vahle, J. L. et al. Skeletal changes in rats given daily subcutaneous injections of recombinant human parathyroid hormone (1-34) for 2 years and relevance to human safety. Toxicol. Pathol. 30, 312-321 (2002).

55. Nakamura, R., Nakamoto, C., Obama, H., Durward, E. \& Nakamoto, M. Structure-function analysis of Nel, a thrombospondin-1-like glycoprotein involved in neural development and functions. J. Biol. Chem. 287, 3282-3291 (2012).

56. Lee, M., Siu, R. K., Ting, K. \& Wu, B. M. Effect of Nell-1 delivery on chondrocyte proliferation and cartilaginous extracellular matrix deposition. Tissue Eng. A 16, 1791-1800 (2010).

57. Askarinam, A. et al. Human perivascular stem cells show enhanced osteogenesis and vasculogenesis with Nel-like molecule I protein. Tissue Eng. A 19, 1386-1397 (2013).

58. Parfitt, A. M. et al. Bone histomorphometry: standardization of nomenclature, symbols, and units. Report of the ASBMR Histomorphometry Nomenclature Committee. J. Bone Miner. Res. 2, 595-610 (1987).

59. Klinck, J. \& Boyd, S. K. The magnitude and rate of bone loss in ovariectomized mice differs among inbred strains as determined by longitudinal in vivo microcomputed tomography. Calcif. Tissue Int. 83, 70-79 (2008).

60. Buie, H. R., Moore, C. P. \& Boyd, S. K. Postpubertal architectural developmental patterns differ between the L3 vertebra and proximal tibia in three inbred strains of mice. J. Bone Miner. Res. 23, 2048-2059 (2008).

61. Bouxsein, M. L., Myers, K. S., Shultz, K. L., Donahue, L. R., Rosen, C. J. \& Beamer, W. G. Ovariectomy-induced bone loss varies among inbred strains of mice. J. Bone Miner. Res. 20, 1085-1092 (2005).

62. Kwak, J. et al. NELL-1 injection maintains long-bone quantity and quality in an ovariectomy-induced osteoporotic senile rat model. Tissue Eng. A 19, 426-436 (2013).

\section{Acknowledgements}

We thank the following individuals and offices for their expertise: TPCL and Surgical Pathology divisions of the UCLA Department of Pathology and Laboratory Medicine, the UCLA Statistical Consulting Center, the Crump Institute for Molecular Imaging, the BioDent testing facility, Drs Ender, Lassman, Nelson, Said, Pang, Lusis, Davis, Stout, Pereira, Prikhodko and Tetradis. We thank the following individuals for their excellent technical assistance: Drs Chiang, Chung, Kim, Hu, Park, Scott, Valesco, Zara and Zhang, and Mr/Ms Askarinam, Chang, Chawan, Covarrubias, Ghiam, Hakim, James, Khadarian, Kim, Le, A. Nguyen, V. Nguyen, Pan, Schnitzer and Siu. This work was supported by the CIRM Early Translational II Research Award TR2-01821, NIH/NIDCR (grants R01 AR066782-01 and R01 AR061399-01A1), and T32 training fellowship to A.W.J. (5T32DE007296-14).

\section{Author contributions}

A.W.J., J.S., X.Z., K.T. and C.S. jointly conceived the study. A.W.J., J.S., X.Z., H.B.S., A.S.T., J.S.A., K.T. and C.S. designed the study. A.W.J., J.S., X.Z., G.A., R.G., J.K., L.J., B.B., A.S.T. and H.B.S. performed the experiments and analysed the data. B.B., C.T.C., A.S.T., H.B.S., K.L., J.S.A., K.T., C.S. and B.W. donated supplies and/or funding. All authors contributed extensively to the work presented. All authors discussed the results and implications and commented on the manuscript at all stages.

\section{Additional information}

Supplementary Information accompanies this paper at http://www.nature.com/ naturecommunications

Competing financial interests: X.Z., B.W., K.T. and C.S. are inventors of NELL-1-related patents. X.Z., B.W., K.T. and C.S. are founders and/or board members of Bone Biologics Inc., which sublicenses NELL-1 patents from the UC Regents, and also hold equity in the company. C.T.C. is an inventor of NELL-1-related patents filed from ORNL and is a founder of NellOne Therapeutics Inc., which licenses NELL-1-related patent applications from ORNL. The remaining authors declare no competing financial interests.

Reprints and permission information is available online at http://npg.nature.com/ reprintsandpermissions/

How to cite this article: James, A. W. et al. NELL-1 in the treatment of osteoporotic bone loss. Nat. Commun. 6:7362 doi: 10.1038/ncomms8362 (2015).

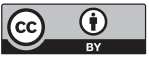

This work is licensed under a Creative Commons Attribution 4.0 International License. The images or other third party material in this article are included in the article's Creative Commons license, unless indicated otherwise in the credit line; if the material is not included under the Creative Commons license, users will need to obtain permission from the license holder to reproduce the material. To view a copy of this license, visit http://creativecommons.org/licenses/by/4.0/ 